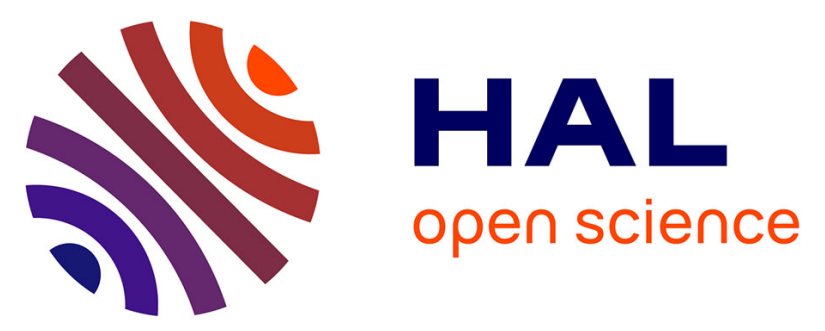

\title{
An alternative to inverse dynamics joint torques estimation in human stance based on a Takagi-Sugeno unknown inputs observer in the descriptor form
}

Kevin Guelton, Sebastien Delprat, Thierry-Marie Guerra

\section{To cite this version:}

Kevin Guelton, Sebastien Delprat, Thierry-Marie Guerra. An alternative to inverse dynamics joint torques estimation in human stance based on a Takagi-Sugeno unknown inputs observer in the descriptor form. Control Engineering Practice, 2008, 16 (12), pp.1414-1426. 10.1016/j.conengprac.2008.04.002 . hal-00793233

\section{HAL Id: hal-00793233 \\ https://hal.science/hal-00793233}

Submitted on 21 Feb 2013

HAL is a multi-disciplinary open access archive for the deposit and dissemination of scientific research documents, whether they are published or not. The documents may come from teaching and research institutions in France or abroad, or from public or private research centers.
L'archive ouverte pluridisciplinaire $\mathbf{H A L}$, est destinée au dépôt et à la diffusion de documents scientifiques de niveau recherche, publiés ou non, émanant des établissements d'enseignement et de recherche français ou étrangers, des laboratoires publics ou privés. 


\title{
AN ALTERNATIVE TO INVERSE DYNAMICS JOINT TORQUES \\ ESTIMATION IN HUMAN STANCE BASED ON A TAKAGI-SUGENO \\ UNKNOWN-INPUTS OBSERVER IN THE DESCRIPTOR FORM
}

\author{
Kevin GUELTON*, Sébastien DELPRAT** and Thierry-Marie GUERRA** \\ *CReSTIC, EA3804, University of Reims \\ Moulin de la House BP1039, 51687 Reims Cedex 2, France \\ Tel : (+33) 326918386 fax : (+33) 326913106 \\ **LAMIH, UMR CNRS 8530, University of Valenciennes \\ Le Mont Houy, 59313 Valenciennes Cedex 9, France \\ Tel : (+33) 327511487 fax : (+33) 327511318 \\ E-Mail : kevin.guelton@univ-reims.fr
}

Abstract: An alternative to inverse-dynamics joint-torques estimation in human stance is proposed. This alternative is based on unknown-input observers which, from measurements of the angular positions, allow real-time estimation of joint torques and angular velocities. To design the aforementioned observer, a nonlinear state-space dynamical descriptor model is proposed. The descriptor model is then written into the form of a Takagi-Sugeno (T-S) model, from which a T-S observer in the descriptor form is designed. Then, convergence of the estimation errors (in position, velocities and torques) is established using a quadratic Lyapunov function through LMI conditions. Finally, the proposed approach is successfully compared with commonly used inverse-dynamics jointtorques estimation. 
Keywords: Human Torque Estimation, Human Standing, Double Inverted Pendulum, Inverse-Dynamics, Descriptor Systems, Fuzzy Observers.

\section{INTRODUCTION}

Biomechanics applies the procedures and knowledge of many different sciences and disciplines in original ways. In this article, concepts drawn from automatic control theory and computer science are employed to deal with a biomechanical problem: the evaluation of joint torques in human standing. Realizing a movement or staying steady is an easy task for healthy people but it could prove to be a difficult work for those suffering from strokes or postural disorders. Improving movement or postural capabilities is possible only when a good diagnosis is made. Thus, since the myoskeletal system can be regarded as a dynamical one where segment positions and trajectories are the system outputs and joint torques are the inputs, postural or movement disorders can be more accurately evaluated by clinicians when both are well measured. Joint torques are classically estimated in biomechanics from motion capture and measurement of the ground reaction force. The well known "bottom-up" inverse-dynamics method is then used to solve Newton-Euler equations for each segment from the ground (where external forces are measured) to the top, one after another (Winter, 1990). The major drawback of this technique is that measurement errors are reported successively from the bottom to the top, leading to uncertainties on the estimation of joint torques (Zajac, 1993; Cahouët and Amarantini, 2002). These measurement errors are partially due to the confrontation of two heterogeneous captors, the force plate and the motion capture device, and especially with 
the difficulties to make the two captor frames perfectly coincide in experimental studies (McCaw and De Vita, 1995). An objective to define an efficient joint torques estimation technique is to reduce the number of captors to its minimum, i.e. the motion capture device. Another source of error is the need of computing velocities from the segments positions, which makes the inverse-dynamics joint torques estimation highly noisesensitive and ruins real-time estimations (Zajac, 1993; Hatze, 2001). In this paper, an alternative to inverse dynamics is proposed by the use of a nonlinear unknown-input observer that allows the real-time estimation of both the joint torques and the angular velocities from angular positions measurement. Based on a nonlinear state-space descriptor dynamical model of human stance, presented in the next section, the objective is to depict a convenient way to design a nonlinear observer allowing estimation of joint torques in human stance with good accuracy. Among nonlinear control theory, Takagi-Sugeno (T-S) fuzzy model-based approach has nowadays become popular since it has shown its efficiency to control complex nonlinear systems in a wide range of application area, see e.g (Khiar et al., 2007; Liu, 2007; Abdelazim and Malik, 2005; Bernal et al., 2006). Indeed, Takagi and Sugeno have proposed a fuzzy model to describe nonlinear models (Takagi and Sugeno, 1985) as a collection of linear time invariant (LTI) models blended together with nonlinear functions. Conversely, T-S descriptors have been introduced by (Taniguchi et al., 2001) and some recent results have dealt with controller design (Bouarar et al., 2007) or observer design for descriptor systems (Marx et al., 2007). Nevertheless, in the latter, the proposed observer is not strictly in the descriptor form leading to less applicability to mechanical systems. Therefore, stability conditions for T-S observers in the descriptor form are provided in section 3. Then, a fuzzy T-S unknown-input observer in descriptor form is derived from the proposed nonlinear model of human standing in 
section 4. Finally, based on simulations and experimental results, comparisons between the obtained T-S unknown-inputs observer results and the ones obtained with the commonly used in biomechanics inverse dynamics approaches will be discussed.

\section{DOUBLE INVERTED PENDULUM MODELING OF HUMAN}

\section{STANCE}

When an individual is quietly standing (i.e. without voluntary movement) in the sagittal plane, knees and neck movements are slower than ankles and hip movements. Thus, depending on the environment and certain pathological assumptions, two main sway strategies have been experimentally observed: the "ankle" strategy and the "hip" strategy (Nashner and McCollum, 1985). When a quietly standing individual is mainly oscillating around the ankle, the sway strategy is called the ankle strategy and, when he is mainly oscillating around the hip, the sway strategy is called the hip strategy. According to that, a planar double inverted pendulum has been chosen to model the human body in the sagittal plane, figure 1. The lower pendulum oscillates around point $A$ at the ankle, and the upper pendulum around point $H$ at the hip.

This model is based on two assumptions:

- both inverted pendulum segments are rigid and connected by hinge joints

- the neuromuscular system, i.e. the muscle actuators and the central nervous system, provides the necessary joint torques $u_{1}$ at the ankle and $u_{2}$ at the hip to stabilize standing.

These torques are the inputs of the double inverted pendulum model, and the associated angular positions are its outputs. In figure 1, segment 1 represents the lower limbs, not 
including the feet; segment 2 represents the trunk, head and upper limbs. The inertial characteristics $m_{1}, m_{2}, I_{1}, I_{2}$ and $K$ for both segments 1 and 2 can be estimated using anthropometric tables (Winter, 1990). $\theta_{1}$ and $\theta_{2}$ are the generalized angular coordinates of segments 1 and 2 with respect to the vertical axis.

This double inverted pendulum dynamical model can be obtained using the Lagrange's equation and is given by:

$M\left(\theta_{1}(t), \theta_{2}(t)\right) \ddot{y}(t)+S\left(\theta_{1}(t), \theta_{2}(t), \theta_{1}(t), \dot{\theta}_{2}(t)\right) \dot{y}(t)+G\left(\theta_{1}(t), \theta_{2}(t)\right) y(t)=R u(t)(1)$

with $u(t)=\left[\begin{array}{lll}u_{1}(t) & u_{2}(t)\end{array}\right]^{T}$ the torques vector, $y(t)=\left[\begin{array}{ll}\theta_{1}(t) & \theta_{2}(t)\end{array}\right]^{T}$ the positions vector, $M\left(\theta_{1}(t), \theta_{2}(t)\right)=\left[\begin{array}{cc}a & c \cos \left(\theta_{1}(t)-\theta_{2}(t)\right) \\ c \cos \left(\theta_{1}(t)-\theta_{2}(t)\right) & b\end{array}\right]$ the inertia matrix, $S\left(\theta_{1}(t), \theta_{2}(t), \dot{\theta}_{1}(t), \dot{\theta}_{2}(t)\right)=\left[\begin{array}{cc}0 & c \dot{\theta}_{2}(t) \sin \left(\theta_{1}(t)-\theta_{2}(t)\right) \\ -c \dot{\theta}_{1}(t) \sin \left(\theta_{1}(t)-\theta_{2}(t)\right) & 0\end{array}\right]$ the anti-symmetric matrix, $G\left(\theta_{1}(t), \theta_{2}(t)\right)=\left[\begin{array}{cc}d \frac{\sin \theta_{1}(t)}{\theta_{1}(t)} & 0 \\ 0 & e \frac{\sin \theta_{2}(t)}{\theta_{2}(t)}\end{array}\right]$ the gravitational matrix, $R=\left[\begin{array}{cc}1 & -1 \\ 0 & 1\end{array}\right]$ the matrix linking joint torques to generalized torques and the constant parameters $\quad a=I_{1}+m_{1} K^{2} L_{1}^{2}+m_{2} L_{1}^{2}, \quad b=I_{2}+m_{2} L_{2}^{2}, \quad c=m_{2} L_{1} L_{2}$, $d=\left(m_{2}+m_{1} K\right) g L_{1}$ and $e=m_{2} g L_{2}$.

Considering $x^{T}=\left[\begin{array}{llll}\theta_{1} & \theta_{2} & \dot{\theta}_{1} & \dot{\theta}_{2}\end{array}\right]$ a state vector of the system, (1) can be written as the following nonlinear state space descriptor: 
$\left\{\begin{array}{l}E(x(t)) \dot{x}(t)=A(x(t)) x(t)+B u(t) \\ y(t)=C x(t)\end{array}\right.$

with: $E(x(t))=\left[\begin{array}{cc}I & 0 \\ 0 & M\left(\theta_{1}, \theta_{2}\right)\end{array}\right], A(x(t))=\left[\begin{array}{cc}0 & I \\ G\left(\theta_{1}, \theta_{2}\right) & -S\left(\theta_{1}, \theta_{2}, \dot{\theta}_{1}, \dot{\theta}_{2}\right)\end{array}\right], B=\left[\begin{array}{l}0 \\ R\end{array}\right]$ and $C=\left[\begin{array}{ll}I & 0\end{array}\right]$

\section{T-S FUZZY DESCRIPTOR MODELS AND OBSERVERS}

\section{T-S modelling:}

Let us consider the generic state-space representation of a nonlinear descriptor model as:

$\left\{\begin{array}{l}E(x(t)) \dot{x}(t)=A(x(t)) x(t)+B(x(t)) u(t) \\ y(t)=C(x(t)) x(t)\end{array}\right.$

where $x(t) \in \square^{n}$ is the state vector, $u(t) \in \square^{m}$ the input vector and $y(t) \in \square^{p}$ the outputs vector. For simplicity, one considers the problem always well defined, i.e. $E(x(t))$ is regular for each $x(t) \in \square^{n}$.

A fuzzy Takagi-Sugeno representation in a descriptor form leads to a collection of LTI models in descriptor form blended together with nonlinear functions (Taniguchi et al., 2000):

$$
\left\{\begin{array}{l}
\sum_{k=1}^{e} v_{k}(z(t)) E_{k} \dot{x}(t)=\sum_{i=1}^{r} h_{i}(z(t))\left(A_{i} x(t)+B_{i} u(t)\right) \\
y(t)=\sum_{i=1}^{r} h_{i}(z(t)) C_{i} x(t)
\end{array}\right.
$$

where $e$ and $r$ are respectively the number of fuzzy sets for the left and the right part of the state equation (4). $z(t)$ is the premise vector depending on the state variables 
$\theta_{1}(t), \theta_{2}(t), \dot{\theta}_{1}(t) \quad$ and $\quad \dot{\theta}_{2}(t)$. Functions $\quad h_{i}(z(t)) \geq 0, \quad i \in\{1, \ldots, r\}, \quad v_{k}(z(t)) \geq 0$, $k \in\{1, \ldots, e\}$ satisfy the convex sum property, i.e. $\sum_{k=1}^{e} v_{k}(z)=1$ (resp. $\left.\sum_{i=1}^{r} h_{i}(z)=1\right) . E_{k}$, $A_{i}, B_{i}$ and $C_{i}$ are the matrices defining each $e \times r$ LTI descriptors that compose the T-S descriptor.

Recall that there is a systematic way to go from the nonlinear descriptor (3) to one of its T-S representation (4). This way is called the sector nonlinearity approach (Tanaka and Wang, 2001). In that case, the T-S descriptor (4) matches exactly the nonlinear model (3) in a compact set of the state variables. Note that infinity of T-S models can represent a single nonlinear model (Taniguchi et al., 2001). At last, the number of linear models obtained is directly linked to the number of the considered nonlinear terms. If there are $n_{L}$ nonlinear terms involved in the sector nonlinearity approach, then the number of linear models of the TS model is $2^{n_{L}}$. Of course, the greater the number of linear models the more conservative the results (see Tanaka and Wang 2001 and Sala et al. 2005 and references therein). Due to this the descriptor model of human stance (2) can be written in a T-S descriptor using the well-known sector nonlinearity approach form considering the nonlinear functions contained in the matrices $E(x(t))$ and $A(x(t))$. These functions are:

$$
\begin{aligned}
& \omega\left(\theta_{1}, \theta_{2}\right)=\cos \left(\theta_{1}-\theta_{2}\right) \in[-1,1] \\
& \eta_{i}\left(\theta_{i}\right)=\frac{\sin \left(\theta_{i}\right)}{\theta_{i}} \in[\underline{\eta}, 1], \quad i=1,2 \\
& \left.\eta_{3}\left(\theta_{1}, \theta_{2}, \dot{\theta}_{1}\right)=\dot{\theta}_{1} \sin \left(\theta_{1}-\theta_{2}\right) \in\right]-\infty,+\infty[ \\
& \left.\eta_{4}\left(\theta_{1}, \theta_{2}, \dot{\theta}_{2}\right)=\dot{\theta}_{2} \sin \left(\theta_{1}-\theta_{2}\right) \in\right]-\infty,+\infty[
\end{aligned}
$$

with $\underline{\eta} \approx-0.2172$ corresponding to the minimum of the function $\eta_{i}($.$) over \square$. 
Thus a TS model can be derived matching exactly the nonlinear model in a compact set of the state vector (with limited rates) using $2^{5}=32$ linear models.

Note that the functions $\eta_{3}\left(\theta_{1}, \theta_{2}, \dot{\theta}_{1}\right)$ and $\eta_{4}\left(\theta_{1}, \theta_{2}, \dot{\theta}_{2}\right)$, due to the Coriolis effect, are physiologically limited and can be neglected because of the small range of the velocities in human standing. Moreover, in the case of T-S state estimation it is easier to cope with a premise vector $z(t)$ depending only on the measured variables $y(t)$ (Guerra et al., 2006).

Both points will be discussed further with the experimental results presentation. Thus, in the sequel a restricted T-S descriptor with $2^{3}=8$ linear models will be constructed by eliminating the nonlinearities $\eta_{3}(\cdot)$ and $\eta_{4}(\cdot)$.

To obtain each LTI models and the membership functions composing a T-S model matching (2), the following sector nonlinearity transformation is employed:

$$
\begin{aligned}
& \omega\left(\theta_{1}, \theta_{2}\right)=\cos \left(\theta_{1}-\theta_{2}\right)=\underbrace{\frac{1-\omega\left(\theta_{1}, \theta_{2}\right)}{2}}_{v_{1}\left(\theta_{1}, \theta_{2}\right)} \times(-1)+\underbrace{\frac{\omega\left(\theta_{1}, \theta_{2}\right)+1}{2}}_{v_{2}\left(\theta_{1}, \theta_{2}\right)} \times 1 \\
& \eta_{i}\left(\theta_{i}\right)=\frac{\sin \left(\theta_{i}\right)}{\theta_{i}}=\underbrace{\frac{1-\eta_{i}\left(\theta_{i}\right)}{1-\eta}}_{\underline{w}_{i 1}(z)} \times \underline{\eta}+\underbrace{\frac{\eta_{i}\left(\theta_{i}\right)+\underline{\eta}}{1-\underline{\eta}}}_{w_{i 2}(z)} \times 1, i=1,2 \text { and } \underline{\eta} \approx-0.2217
\end{aligned}
$$

Thus, (9) and (10) allow defining the membership functions:

$$
\begin{aligned}
& v_{1}\left(\theta_{1}, \theta_{2}\right)=\frac{1-\cos \left(\theta_{1}-\theta_{2}\right)}{2} \text { and } v_{2}\left(\theta_{1}, \theta_{2}\right)=\frac{\cos \left(\theta_{1}-\theta_{2}\right)+1}{2} \\
& h_{1}\left(\theta_{1}, \theta_{2}\right)=w_{11}\left(\theta_{1}\right) w_{21}\left(\theta_{2}\right)=\frac{\left(1-\eta_{1}\left(\theta_{1}\right)\right)\left(1-\eta_{2}\left(\theta_{2}\right)\right)}{(1-\underline{\eta})^{2}} \\
& h_{2}\left(\theta_{1}, \theta_{2}\right)=w_{12}\left(\theta_{1}\right) w_{22}\left(\theta_{2}\right)=\frac{\left(1-\eta_{1}\left(\theta_{1}\right)\right)\left(\eta_{2}\left(\theta_{2}\right)+\underline{\eta}\right)}{(1-\underline{\eta})^{2}}
\end{aligned}
$$


$h_{3}\left(\theta_{1}, \theta_{2}\right)=w_{11}\left(\theta_{1}\right) w_{22}\left(\theta_{2}\right)=\frac{\left(1-\eta_{1}\left(\theta_{1}\right)\right)\left(\eta_{2}\left(\theta_{2}\right)+\underline{\eta}\right)}{(1-\underline{\eta})^{2}}$

$h_{4}\left(\theta_{1}, \theta_{2}\right)=w_{12}\left(\theta_{1}\right) w_{21}\left(\theta_{2}\right)=\frac{\left(\eta_{1}\left(\theta_{1}\right)+\underline{\eta}\right)\left(1-\eta_{2}\left(\theta_{2}\right)\right)}{(1-\underline{\eta})^{2}}$

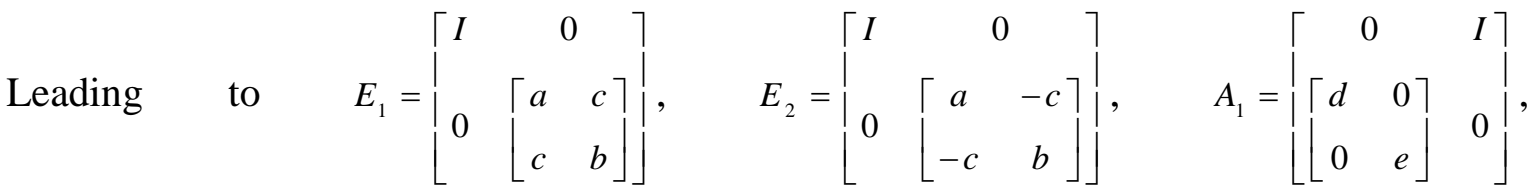

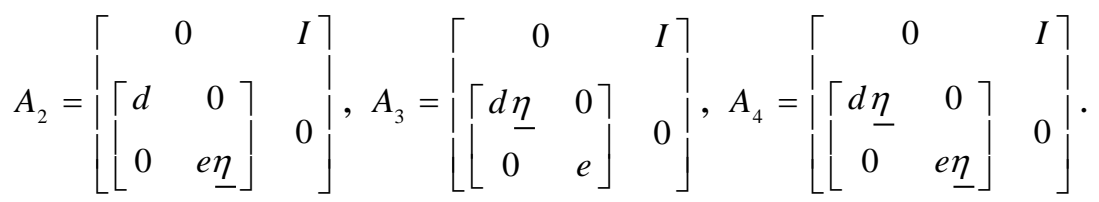

Thus, considering $z^{T}(t)=\left[\begin{array}{ll}\theta_{1} & \theta_{2}\end{array}\right]$, the simplified fuzzy T-S descriptor model of human stance can be expressed as:

$$
\left\{\begin{array}{l}
\sum_{k=1}^{2} v_{k}(z(t)) E_{k} \dot{x}(t)=\sum_{i=1}^{4} h_{i}(z(t)) A_{i} x(t)+B u(t) \\
y(t)=C x(t)
\end{array}\right.
$$

Fuzzy Takagi-Sugeno observer descriptor structure:

Using the general T-S descriptor form (4), it is possible to propose a nonlinear observer based on this form:

$$
\begin{aligned}
& \int \sum_{k=1}^{e} v_{k}(z(t)) E_{k} \dot{\hat{x}}(t)=\sum_{i=1}^{r} h_{i}(z(t))\left[A_{i} \hat{x}(t)+B_{i} u(t)\right] \\
& +\sum_{k=1}^{e} \sum_{i=1}^{r} v_{k}(z(t)) h_{i}(z(t)) K_{i k}(y(t)-\hat{y}(t)) \\
& \hat{y}(t)=\sum_{i=1}^{r} h_{i}(z(t)) C_{i} \hat{x}(t)
\end{aligned}
$$

where $\hat{x}(t)$ and $\hat{y}(t)$ are respectively the estimated state vector and the output vector. $K_{i k}$ are the observer gain matrices. Let us quote that the general case would imply the use of 
$\hat{z}(t)$, i.e. $h_{i}(\hat{z}(t))$ and $v_{k}(\hat{z}(t))$ instead of $h_{i}(z(t))$ and $v_{k}(z(t))$. This general case is harder and very few results are available (Guerra et al., 2006). The purpose of this section is to derive sufficient conditions of convergence for the estimation error of a T-S observer. Summarizing for the present application, choosing an approximation of the nonlinear descriptor model (2) with $2^{3}=8$ LTI models, instead of an exact one with $2^{5}=32$ LTI models (included the neglected functions $\eta_{3}(\cdot)$ and $\left.\eta_{4}(\cdot)\right)$ is a good compromise between model complexity (in terms of LMI resolution) and its reliability with respect to the application.

\section{Estimation error convergence:}

A way to obtain sufficient convergence conditions for T-S fuzzy descriptor observer is to rewrite (4) using the augmented state $x^{*}(t)=\left[x^{T}(t), \dot{x}^{T}(t)\right]^{T}$ :

$$
\left\{\begin{array}{l}
E^{*} \dot{x}^{*}(t)=\sum_{k=1}^{e} \sum_{i=1}^{r} v_{k}(z(t)) h_{i}(z(t)) A_{i k}^{*} x^{*}(t)+\sum_{i=1}^{r} h_{i}(z(t)) B_{i}^{*} u(t) \\
y(t)=\sum_{i=1}^{r} h_{i}(z(t)) B_{i}^{*} x^{*}(t)
\end{array}\right.
$$

where $E^{*}=\left[\begin{array}{ll}I & 0 \\ 0 & 0\end{array}\right], A_{i k}^{*}=\left[\begin{array}{cc}0 & I \\ A_{i} & -E_{k}\end{array}\right], B_{i}^{*}=\left[\begin{array}{c}0 \\ B_{i}\end{array}\right]$ and $C_{i}^{*}=\left[\begin{array}{ll}C_{i} & 0\end{array}\right]$. In a similar way, the fuzzy T-S observer (17) is written as, with $\hat{x}^{*}(t)=\left[\hat{x}^{T}(t), \dot{\hat{x}}^{T}(t)\right]^{T}$ and $K_{i k}^{*}=\left[\begin{array}{c}0 \\ K_{i k}\end{array}\right]$ :

$$
\left\{\begin{aligned}
E^{*} \dot{\hat{x}}^{*}(t)=\sum_{k=1}^{e} \sum_{i=1}^{r} v_{k}(z(t)) h_{i}(z(t)) A_{i k}^{*} \hat{x}^{*}(t)+\sum_{i=1}^{r} h_{i}(z(t)) B_{i}^{*} u(t) & \\
& +\sum_{k=1}^{e} \sum_{i=1}^{r} v_{k}(z(t)) h_{i}(z(t)) K_{i k}^{*}(y(t)-\hat{y}(t)) \\
y(t)=\sum_{i=1}^{r} h_{i}(z(t)) B_{i}^{*} \hat{x}^{*}(t) &
\end{aligned}\right.
$$


Let us define the augmented prediction error as $\tilde{x}^{*}(t)=\left[(x(t)-\hat{x}(t))^{T},(\dot{x}(t)-\dot{\hat{x}}(t))^{T}\right]^{T}$; its derivative can be written as:

$$
E^{*} \dot{\tilde{x}}^{*}(t)=\sum_{k=1}^{e} \sum_{i=1}^{r} \sum_{j=1}^{r} v_{k}(z(t)) h_{i}(z(t)) h_{j}(z(t))\left[A_{i k}^{*}-K_{j k}^{*} C_{i}^{*}\right] \tilde{x}^{*}(t)
$$

The following theorem ensures the convergence of the state reconstruction error for a suitable set of $K_{i k}^{*}$ (Guelton et al., 2006).

Theorem 1: Let us consider the fuzzy descriptor model (20). The convergence of the estimation error is ensured if there exist: $P_{1}=P_{1}^{T}>0, P_{3}$ and $P_{4}$ regular, $K_{i k}$, $k \in\{1, \ldots, e\}, i, j \in\{1, \ldots, r\}, j>i$, such that:

$\Upsilon_{i i}^{k}<0$

$\Upsilon_{i j}^{k}+\Upsilon_{j i}^{k}<0$

with $\Upsilon_{i j}^{k}=\left[\begin{array}{cc}A_{i}^{T} P_{3}+P_{3}^{T} A_{i}-C_{i}^{T} K_{j k}^{T} P_{3}-P_{3}^{T} K_{j k} C_{i} & (*) \\ P_{1}-E_{k}^{T} P_{3}+P_{4}^{T} A_{i}-P_{4}^{T} K_{j k} C_{i} & -E_{k}^{T} P_{4}-P_{4}^{T} E_{k}\end{array}\right]$.

Note that a star $(*)$ in a symmetric matrix indicates a transpose quantity.

Proof: Consider the Lyapunov candidate function $V\left(\tilde{x}^{*}(t)\right)=\tilde{x}^{* T}(t) E^{* T} P \tilde{x}^{*}(t)$, then (20) is stable if:

$E^{* T} P=P E^{*}>0$

and

$\dot{\tilde{x}}^{* T}(t) E^{* T} P \tilde{x}^{*}(t)+\tilde{x}^{*}(t) E^{* T} P \dot{\tilde{x}}^{*}(t)<0$ 
are satisfied.

Consider $P=\left[\begin{array}{ll}P_{1} & P_{2} \\ P_{3} & P_{4}\end{array}\right],(23)$ leads to $P_{1}=P_{1}^{T}>0$ and $P_{2}=0$. Moreover, with (20) and (23), (24) can be rewritten as:

$\left.\tilde{x}^{* T}\left(\sum_{k=1}^{e} \sum_{i=1}^{r} \sum_{j=1}^{r} v_{k}(z(t)) h_{i}(z(t)) h_{j}(z(t))\left(\left[A_{i k}^{*}-K_{j k}^{*} C_{i}^{*}\right]^{T} E^{* T} P+P E^{*}\left[A_{i k}^{*}-K_{j k}^{*} C_{i}^{*}\right]\right)\right)\right) \tilde{x}^{*}<0$

After mathematical development with the matrices defined in (18) and (19), (25) yields:

$$
\sum_{k=1}^{e} \sum_{i=1}^{r} \sum_{j=1}^{r} v_{k}(z(t)) h_{i}(z(t)) h_{j}(z(t))\left[\begin{array}{cc}
A_{i}^{T} P_{3}+P_{3}^{T} A_{i}-C_{i}^{T} K_{j k}^{T} P_{3}-P_{3}^{T} K_{j k} C_{i} & (*) \\
P_{1}-E_{k}^{T} P_{3}+P_{4}^{T} A_{i}-P_{4}^{T} K_{j k} C_{i} & -E_{k}^{T} P_{4}-P_{4}^{T} E_{k}
\end{array}\right]<0
$$

Obviously (26) is satisfied if (21) and (22) hold.

Note that it is straightforward to apply any relaxation schemes found in the literature in addition to the conditions given in theorem 1 (Liu and Zhang, 2003; Tuan et al., 2001). At last, let us point out that conditions (21) and (22) are LMIs in $P_{1}, P_{3}$ and $P_{4}$, i.e. providing that $K_{i k}$ are given. On the contrary, if $K_{i k}$ and $P_{1}, P_{3}, P_{4}$ have to be searched together, it is necessary to render these conditions LMI. Some results are available in (Guelton, 2003; Guerra et al., 2004). Note also that, for the present application, $K_{i k}$ are computed apart using pole placement to ensure some performances, whereas theorem 1 is only used to check the stability.

Comment 1: As stated in (Guerra et al., 2004; Guelton 2003), it can be interesting, in the case of mechanical systems with time varying inertia, to investigate the convergence of an observer based on a descriptor form rather than a "classical" state-space representation. 
Indeed, the descriptor approach appears sometimes to be less conservative than the "classical" approach. Of course in order to solve the descriptor state estimation with the use of classical ODE solvers, $E(x(t))$ have to be invertible. Note that the equation (2) is always well defined as $E(x(t))$ is non singular for each $y(t) \in \square^{n} \times \square^{n}$ since:

$E(x)$ non singular $\Leftrightarrow \forall\left(\theta_{1}, \theta_{2}\right), M\left(\theta_{1}, \theta_{2}\right)$ invertible $\Leftrightarrow \forall\left(\theta_{1}, \theta_{2}\right), \operatorname{det}\left(M\left(\theta_{1}, \theta_{2}\right)\right) \neq 0$.

$\Leftrightarrow \operatorname{det}(M)=\left(m_{2} L_{2}^{2}+I_{2}\right)\left(m_{1} K^{2} L_{1}^{2}+I_{1}+m_{2} L_{1}^{2}\right)-\left(m_{2} L_{1} L_{2} \cos \left(\theta_{1}-\theta_{2}\right)\right)^{2}=0$

$\Leftrightarrow \cos \left(\theta_{1}-\theta_{2}\right)=\sqrt{1+\frac{L_{1}^{2} m_{2} L_{2}^{2}\left(m_{1} K^{2}+I_{2}\right)+I_{1} m_{2} L_{2}^{2}+I_{1} I_{2}+m_{2} L_{1}^{2} I_{1}}{m_{2}^{2} L_{1}^{2} L_{2}^{2}}}$, which obviously is not possible on $\square$ since $\frac{L_{1}^{2} m_{2} L_{2}^{2}\left(m_{1} K^{2}+I_{2}\right)+I_{1} m_{2} L_{2}^{2}+I_{1} I_{2}+m_{2} L_{1}^{2} I_{1}}{m_{2}^{2} L_{1}^{2} L_{2}^{2}}>0$.

To illustrate the benefit of a descriptor approach for some nonlinear models, let us consider an example. With the state vector being also the premise vector $x=z=\left[\begin{array}{ll}x_{1} & x_{2}\end{array}\right]^{T}$ let define:

$E\left(x_{1}, x_{2}\right)=\left[\begin{array}{cc}\frac{1}{1+x_{1}^{2}} & 1 \\ -1 & \frac{1}{1+x_{2}^{2}}\end{array}\right], A=\left[\begin{array}{cc}-4.7 & -4.7 \\ 0.2 & 1.5\end{array}\right]$ and $C\left(x_{2}\right)=\left[\begin{array}{ll}\cos \left(x_{2}\right)+3 & 1\end{array}\right]$.

This is constructed in a way such that classical conditions for fuzzy observer design (Liu and Zhang, 2003) cannot be fulfilled. The observer in a descriptor form will have $2^{3}=8$ LTI models, due to the nonlinear terms $\frac{1}{1+x_{1}^{2}}, \frac{1}{1+x_{2}^{2}}$ and $\cos \left(x_{2}\right)$. Its representation is exact in the whole space $\square^{2}$ as the nonlinearities are bounded. The number of LMI conditions without any relaxation scheme is: $e \cdot r(r+1) / 2=12$. Let us notice that a 
"classical" TS observer will be obtained using $E^{-1}\left(x_{1}, x_{2}\right)$. That leads to consider 4 different nonlinearities, thus $2^{4}=16$ LTI models. What is worse is that the number of LMI conditions is then $r(r+1) / 2=136$ which explains that classical conditions fail.

\section{UNKNOWN INPUT OBSERVER DESIGN}

Let us recall that the primary objective of this study is the joint torques estimation. In human studies, measuring joint torque with external apparatus is ethically outlawed. To overcome this problem, an unknown input observer will be designed under the assumption $\dot{u}(t) \approx 0$. This hypothesis states that the input dynamics is much slower than the observer state dynamics.

Defining the extended vector $x^{e}(t)=\left[\begin{array}{lll}x^{T}(t) & u^{T}(t)\end{array}\right]^{T}$, the T-S descriptor observer (17) reduces to:

$\left\{\begin{array}{l}\sum_{k=1}^{e} v_{k}(z(t)) E_{k}^{e} \dot{\hat{x}}^{e}(t)=\sum_{i=1}^{r} h_{i}(z(t)) A_{i}^{e} \hat{x}^{e}(t)+\sum_{k=1}^{e} \sum_{i=1}^{r} v_{k}(z(t)) h_{i}(z(t)) K_{i k}^{e}(y(t)-\hat{y}(t)) \\ \hat{y}(t)=C^{e} \hat{x}^{e}(t)\end{array}\right.$

with $E_{k}^{e}=\left[\begin{array}{cc}E_{k} & 0 \\ 0 & I\end{array}\right], A_{i}^{e}=\left[\begin{array}{cc}A_{i} & B \\ 0 & 0\end{array}\right], K_{i k}^{e}=\left[\begin{array}{c}K_{i k} \\ 0\end{array}\right]$ and $C^{e}=\left[\begin{array}{ll}C & 0\end{array}\right]$ and matrices $E_{k}, A_{i}, B$ and $C$ defined as in equation (16).

\section{Observer tuning:}

In order to simulate the joint torques estimated by the unknown inputs observer, the whole nonlinear model has to be stabilized. Several ways to ensure the closed loop stability and performances can be investigated, pole placement, quadratic criterion, $H_{\infty}$ attenuation $\ldots$ 
In the case of the proposed application, it can be shown that using a simple linear state feedback control law $u(t)=-F x(t)$ associated with a pole placement technique is enough to reach the prescribed objectives, i.e. stabilizing the model (16) around the erect position, the unstable equilibrium point $\theta_{1}=\theta_{2}=0$ and ensuring a faster system dynamic than the observer with a reasonable behaviour of the state vector signals, see figure 2 .

In the same way for the estimation error convergence some performances can be easily added using extra LMI conditions (Gahinet et al. 1994). In this study, a pole placement technique was used readily giving good results. Of course, the same kind of results can be obtained by introducing, for example, a decay rate on the Lyapunov function or finding an upper bound of a quadratic criterion (see Tanaka and Wang 2001, Sala et al. 2005 and references therein).

Obviously the observer tuning depends on each investigated individual. In this paper, the torques of a 28 year-old male subject, weighting $69.8 \mathrm{Kg}$ and measuring $1.85 \mathrm{~m}$ were investigated. It is the same individual for the parameter tuning, simulation and real-time experiments. Parameters of the double inverted pendulum model of human stance (1), given in table 1, have been obtained using the anthropometric tables given in (Winter, 1990) and optimized as described in (Guelton et al., 2003).

The tuning has been done in simulation on a flexion movement from the erect position $\left(\theta_{1}, \theta_{2}\right)=(0,0)$ to $\left(\theta_{1}, \theta_{2}\right)=(-\pi / 6, \pi / 3)$, depicted in figure 2 , with realistic dynamic considering physiological constraints, i.e. with acceptable velocities and torques for the human capability. As explained previously, a pole placement technique was used. An acceptable compromise was obtained using four poles at -60 for the linear control. For, the T-S observer gains $K_{i k}$, pole placements was made on the linear models 
$E_{k}^{-1}\left(A_{i}-K_{i k} C\right)$ using two dominant poles at -18 and four auxiliary at -55 . As an illustration, the following observer gain matrices relating to the LTI models $(i, k)=(1,1)$ and $(i, k)=(1,2)$ are given:

$K_{11}=\left[\begin{array}{cc}852 & 849 \\ 1369 & 3033 \\ 0.7 & 0.5 \\ 2.5 & 5.7 \\ 21.8 & 39.6 \\ 14.3 & 31.1\end{array}\right], K_{12}=\left[\begin{array}{cc}849 & -848 \\ -1365 & 3023 \\ 0.7 & -0.5 \\ -2.5 & 5.6 \\ -6.7 & 22.4 \\ -14.2 & 31\end{array}\right]$.

The convergence of the state estimation error is ensured with the computed gains $K_{i k}$ if the conditions given in theorem 1 are satisfied. The following solution of theorem 1, obtained using the Matlab LMI Toolbox (Gahinet et al., 1995), ensures the convergence of the estimation error:

$$
P_{1}=\left[\begin{array}{cccccc}
0.0501 & & & & \\
-0.0012 & 0.0553 & & & \\
-0.1481 & -0.0049 & 4.5036 & & \\
0.0013 & -0.1360 & -0.3046 & 6.9666 & \\
-0.0001 & 0.0000 & -0.0000 & 0.0000 & 0.0000 & \\
0.0002 & -0.0011 & 0.0002 & -0.0015 & -0.0000 & 0.0000
\end{array}\right],
$$




$$
P_{4}=\left[\begin{array}{cccccc}
0.0000 & 0.1615 & -0.0004 & -0.0004 & 0.0000 & -0.0039 \\
-1.2115 & 0.0002 & 0.0043 & 0.0215 & 0.0039 & -0.0039 \\
0.0151 & -0.0238 & 0.0008 & -0.0009 & -0.0000 & 0.0006 \\
0.0150 & -0.1184 & 0.0009 & 0.0008 & -0.0001 & 0.0029 \\
-0.0016 & -0.0213 & 0.0000 & 0.0001 & 0.0000 & 0.0005 \\
0.1598 & 0.0216 & 0.0006 & -0.0029 & -0.0005 & 0.0000
\end{array}\right] .
$$

Figure 2 shows the estimate state $\hat{x}(t)$ following trajectories $x(t)$ as well as the two joint torques estimation $\hat{u}(t)$ following the simulated ones $u(t)$ for the observer tuning simulation.

In order to illustrate the benefits of the T-S unknown-inputs observer (27), a comparison with a linear unknown-inputs observer is performed. This linear observer is obtained from the LTI model obtained by the linearization of (2) around the unstable equilibrium point $\theta_{1}=0$ and $\theta_{2}=0$ (erect position); it is written as:

$\left\{\begin{array}{l}E_{L}^{e} \dot{\hat{x}}^{*}(t)=A_{L}^{e} \hat{x}^{*}(t)+K_{L}^{e}(y(t)-\hat{y}(t)) \\ \hat{y}(t)=C^{e} \hat{x}^{e}(t)\end{array}\right.$

with $E_{L}^{e}=\left[\begin{array}{cc}E_{1} & 0 \\ 0 & I\end{array}\right], A_{L}^{e}=\left[\begin{array}{cc}A_{1} & B \\ 0 & 0\end{array}\right], K_{L}^{e}=\left[\begin{array}{c}K_{i k} \\ 0\end{array}\right]$ and $C^{e}=\left[\begin{array}{ll}C & 0\end{array}\right]$.

$K_{L}^{e}$ is chosen using the same pole placement as the nonlinear observer. Note that it corresponds to one of the LTI models of the T-S descriptor, i.e. $v_{1}(\cdot)=1$ and $h_{1}(\cdot)=1$. To point out the behavioural differences, "large" angles are used in order to not fulfil the linear approximation. A complex movement has been set up to reach and outrun human standing physiological limits, see figure 3.

From $t=10 \mathrm{~s}$, the error of the linear observer becomes significant since the angular positions are large. Thus, low angles hypothesis is not respected and the linear observer failed to estimate the inputs. On the other hand, the error of the fuzzy observer is 
acceptable except for the fastest transients of the joint torques, at $t=0.5 \mathrm{~s}$ and $t=5 \mathrm{~s}$. Of course this is due to the observer dynamic. Nevertheless, note that these simulated torques are not physiologically possible with respect to the human capabilities.

Comment 2: Recall that the nonlinearities $\eta_{3}\left(\theta_{1}, \theta_{2}, \dot{\theta}_{1}\right)$ and $\eta_{4}\left(\theta_{1}, \theta_{2}, \dot{\theta}_{2}\right)$ have been removed to obtain the T-S model. For the whole set of simulations, these neglected terms contributions remain always lower than $1 \%$ except for fast transient that are not physiologically possible. This explains the choice made.

\section{EXPERIMENTAL RESULTS}

\section{Experimental protocol:}

Movements were captured at a frequency of $120 \mathrm{~Hz}$ using an AMTI force-plate synchronized with a VICON 612 motion capture device (Figure 4). The 3-dimensional reconstruction error was evaluated at $0.6 \mathrm{~mm}$. Reflective markers define fourteen segments of the subject's body. Based on the measured positions of these fourteen body segments, and through the use of anthropometric tables (Winter, 1990) associated with an optimization algorithm (Guelton et al., 2003), the centre of mass and the inertia of both parts of the double inverted pendulum model in the sagittal plane were determined. The subject was asked to stay barefooted on the force plate and two movements were captured. The first one was in quiet standing (QS) during 30s and the second one was a combined trunk flexion/extension (CTE) that disturbed the subject's equilibrium, provoking ankle and hip oscillations in the sagittal plane. 


\section{Linear vs nonlinear observer experimental results:}

From data collected by the motion capture system, the linear and the nonlinear unknown inputs observers have been used to estimate the subject's angular velocities and torques in standing. These results will be compared to the ones obtained from the "global inverse dynamics" method (Winter, 1990) from the motion equation (1) that can be rewritten as:

$\left\{\begin{array}{l}u_{1}=a \ddot{\theta}_{1}+c \ddot{\theta}_{2} \cos \left(\theta_{1}-\theta_{2}\right)+c \dot{\theta}_{2}^{2} \sin \left(\theta_{1}-\theta_{2}\right)-d \sin \theta_{1}+u_{2} \\ u_{2}=c \ddot{\theta}_{1} \cos \left(\theta_{1}-\theta_{2}\right)+b \ddot{\theta}_{2}-c \dot{\theta}_{1}^{2} \sin \left(\theta_{1}-\theta_{2}\right)-e \sin \theta_{2}\end{array}\right.$

with constant parameters $\quad a=I_{1}+m_{1} K^{2} L_{1}^{2}+m_{2} L_{1}^{2}, \quad b=I_{2}+m_{2} L_{2}^{2}, \quad c=m_{2} L_{1} L_{2}$, $d=\left(m_{2}+m_{1} K\right) g L_{1}, e=m_{2} g L_{2}$ and those given in table 1.

To be computed, this method needs the same measurement as the observers, i.e. the angular positions $\left[\begin{array}{ll}\theta_{1} & \theta_{2}\end{array}\right]$, as well as the numerical computation of the angular velocities $\left[\begin{array}{ll}\dot{\theta}_{1} & \dot{\theta}_{2}\end{array}\right]$ and accelerations $\left[\ddot{\theta}_{1} \ddot{\theta}_{2}\right]$. A particular attention has been made to both numerical derivative and filtering processes. According to (Carpenter et al., 2001), $90 \%$ of the spectral power density of the centre of mass excursion is situated between 0 and $6 \mathrm{~Hz}$. Thus, for noise attenuation, both video and force-plate (needed in the following results) data were filtered through a fourth-order zero-phase lag filter with a cut-off frequency of $6 \mathrm{~Hz}$ (Gustafsson, 1996; Mitra, 2001). However, although this cut-off frequency is appropriate for angular position, it is not appropriate for the estimation of successive derivatives. Unfortunately, if angular velocity and acceleration are estimated using a $6 \mathrm{~Hz}$ filtered angular position, the obtained velocity and acceleration measurements may not represent $90 \%$ of the spectrum density of the real angular velocity and acceleration. The aim of this study is not to propose a generic method to overcome this problem; one can only emphasize that particular attention must be paid to the filtering process. It appears 
quite suitable estimating the derivatives using a measured signal, and then filtering the angular velocity and acceleration thus obtained. $\left[\begin{array}{ll}\dot{\theta}_{1} & \dot{\theta}_{2}\end{array}\right]$ and $\left[\begin{array}{ll}\ddot{\theta}_{1} & \ddot{\theta}_{2}\end{array}\right]$ were obtained using the central difference technique on $\left[\begin{array}{ll}\theta_{1} & \theta_{2}\end{array}\right]$ ( Nougier, 2001).

The results for the QS experiment corresponds to small positions and velocities balancing around the equilibrium point. Of course, the linear assumption being nearly true, whatever the method is, the results remain very close and are not presented.

Figure 5 shows the state estimation for the CTE experiment where high angular amplitudes are observed. In the same way as the simulation results, the linear observer provides incorrect estimations when the subject performs a movement that is far from the equilibrium point. To confirm this point, the detailed estimations of the ankle torques $u_{1}$ between $t=5 \mathrm{~s}$ and $t=15 \mathrm{~s}$ are presented figure 6 . Between $t=7 \mathrm{~s}$ and $t=9 \mathrm{~s}$, the linear observer provides no satisfactory estimation. Note that due to the observer dynamic (usable for real-time estimation) a "delay" can be noticed.

As an additional explanation to the linear observer discordance, figure 7 shows the time evolution of the nonlinear membership function $v_{1}(x(t))$ obtained from the nonlinear term $\omega(y)=\cos \left(\theta_{1}-\theta_{2}\right)$ included in the inertia matrix (see model (1) and (16)). Recall that the linear observer corresponds to one of the LTI models of the T-S model, i.e. $v_{1}(y)=1$ and $h_{1}(y)=1$. Thus, when the inertia of the whole body varies with high angular amplitudes $v_{1}(x(t))$ is going far from 1 and the linear observer fails to give good results.

\section{Nonlinear observer vs classical inverse dynamics approaches:}

In biomechanical studies, three inverse-dynamics approaches are often used (Cappozzo and Pedotti, 1975; Winter, 1990): 
- The first one, called "global inverse dynamics", has been previously employed. It is based on the whole body motion equations, i.e. the formal relation linking the joint torques to the angular positions, velocities and accelerations (Zajac and Gordon, 1989). This approach requires the angular positions, velocities and acceleration measurements and/or estimations to compute joint torques.

- The second one is called the "top-down" inverse dynamics and also needs the angular positions, velocities and acceleration measurements and/or estimations to compute joint torques. Computing joint torques follows an iterative process illustrated in figure 8 . In this way, the dynamical equilibrium conditions, given by the Newton-Euler equations, are applied successively to each body segments in order to obtain successively joint torques from the terminal segment to the one in contact with the external environment. This technique is sensitive to noise measurement since the uncertainties due to velocities and accelerations computation are transmitted successively to joint torques estimations (Zajac, 1993). This entails increasing errors related to the iteration number (Challis and Kerving, 1996).

- The third, commonly used in biomechanics, is called "Bottom-up" inverse dynamics. It is based on the same iterative process as the "Top-down" technique but consists in solving the Newton-Euler equation from the body segment in contact with the external environment to the last one (figure 8). Thus, in addition to angular positions, velocities and accelerations measurements and/or computations, the "Bottom-up" technique also requires the external forces measurements. This method is less sensitive to noise measurements than the "Top-down" method since the external force measurement brings an additional knowledge to the angular accelerations (Zajac, 1993). Nevertheless, a new error due to the different measurement apparatus frame positioning is introduced 
in the iterative process (McCaw and De Vita, 1995). Moreover, the external forces measurements contribute to an overestimation that entails the emergence of residual forces and torques. These appear solving the last body segment where the dynamical equilibrium conditions are consequently not verified (Challis and Kerving, 1996; Cahouët and Amarantini, 2002; Hatze, 2002).

The «Bottom-up» and the «Top-down» inverse dynamics approaches are supposed to provide a good estimation at their first iteration. Indeed, the ankle torques computed from the "Bottom-up" technique as well as the hip torques computed with the "Top-down" technique are supposed to provide good estimations (Cappozzo and Pedotti, 1975; Challis and Kerwin, 1996; Hatze, 2002). The detailed formulations associated to these methods applied to the double inverted pendulum model of human stance are presented in appendix1.

Figure 9 (a) and (b) show respectively the results obtained from the three inverse dynamics approaches and the T-S unknown-inputs observer for the CTE experiment at the ankle and at the hip. As expected, the T-S observer and the "global inverse dynamics" provide similar results. Effectively, these two approaches take into account the global nonlinear model (1). As expected, for the "bottom-up" technique (resp. "Top-down") the results on the hip (resp. ankle) are not satisfactory.

In the ideal situation, if the measurements are perfectly homogeneous, "Top-down", "Bottom-up" and "Global" inverse dynamics should provide the same results. Putting into relation data obtained from two different apparatus (force-plate and optoelectronic system) leads to uncertainties due to the intrinsic calibration of each apparatus. This point partially explains the residual hip torques emergence presented in figure 10. These residual torques correspond to the difference between the hip torques estimated by the "Bottom-up" and the 
"Top-down" techniques. In the CTE experiment, they lead to a mean error value of $4,5 \mathrm{Nm}$ and a maximal error up to $13,5 \mathrm{Nm}$ meaning $14,6 \%$ of the whole amplitude variation of the hip torques $u_{2}$.

In the case of the QS experiment, the same conclusion can be done with respect to the ankle and hip joint torques estimations that are respectively presented in figure 11 (a) and (b). The residual hip torques due to the "Bottom-up" approach have a mean value of $4,1 \mathrm{Nm}$ and leads to a maximal error of $5,2 \mathrm{Nm}$ that corresponds to the whole amplitude variation of the hip torques. Moreover, note that concerning the ankle torques, the "Bottom-up" approach leads to discordances despite the fact that it is supposed to be more accurate than the "Top-down" approach. The overestimation effect associated with the "Bottom-up" approach is "amplified" as the studied movement is small.

Unless using an internal measurement apparatus that is ethically outlaw in human studies, there is not any measurement reference available to claim that using an unknown input observer is a better method to compute joint torques than using inverse dynamics. Nevertheless, some elements show its usefulness, for instance the nonlinear unknown input observer always provides close results to the "Global" inverse dynamics approach. Moreover, the ankle and hip torques observer estimation are also close respectively to the "Bottom-up" and the "Top-down" inverse dynamics technique in the CTE experiment. Regarding to the QS experiment, the commonly used "Bottom-up" and "Top-down" inverse dynamics approach in biomechanics have shown their limits with respect to small movements whereas the observer results remain close to the "global" inverse dynamics technique.

The main advantage of the observer based estimation in comparison to the "global inverse dynamics" is real-time capability since they both provide similar results. Indeed, observers 
are classically used in state-feedback control for online state estimation when the whole state vector is not available. In the present study case, the observer allows both the real time estimation of joint torques and angular velocities from the only measurement of the angular positions. Another convenient feature of observer based estimation is its capacity to filter noise measurements by a convenient gain tuning. On the contrary, inversedynamics-based estimation techniques need, when only the positions are measured, the computation of angular velocities and accelerations. Then, in order to obtain relevant estimation, special attention has to be made on the filtering and derivative processes, which makes inverse dynamics real time capability hazardous.

Finally, when a dynamical model can be easily obtained and due to the fact that it only requires the segments positions measurements, one may assume that the use of an unknown-inputs observer is a suitable alternative to the use of inverse dynamics techniques in biomechanical studies.

\section{CONCLUSION}

A nonlinear model of human standing was proposed based on a double inverted pendulum. Then, a fuzzy Takagi-Sugeno unknown-inputs observer in descriptor form was designed to estimate both the joint torques and the velocities from only the angular position measurements. To establish the interest of designing such a nonlinear observer, the results were compared successfully in simulation to a linear observer. An experimental study has shown that using an unknown-input observer is a more suitable method to compute joint torques than using inverse dynamics when a dynamical model is available. Moreover, classical inverse dynamics methods need a careful estimation of velocities and 
accelerations that are not easy when real time joint torques estimation is expected. Consequently, in further study, the use of an unknown-inputs observer for real time human balance rehabilitation based on joint torques biofeedback will be possible.

\section{ACKNOWLEDGMENTS:}

This work was supported in part by the "Nord Pas-de-Calais" Region and the FEDER (European Funds of Regional Development) under the AUTORIS project and by the "Région Champagne-Ardennes" within the framework of the CPER SYS-REEDUC. The authors would like to thanks Mr Bernal for his help within spelling and grammar and $\mathrm{Mr}$ Sayfi-Nybien for his supporting comments within this study.

\section{APPENDIX 1: «TOP-DOWN » AND «BOTTOM-UP » ANKLE AND HIP JOINT TORQUES ESTIMATIONS IN STANDING}

Notations : $F$ denotes the reaction force, $P$ denotes the gravitational force, $M_{F, A}$ denotes the momentum due to the force $F$ at the point $A$ and $G$ denotes the centre of gravity. Subscripts $p, 1$ and 2 are representing respectively the feet, the lower pendulum and the upper pendulum. Indexes $x$ and $z$ denote respectively the horizontal and the vertical component of a vector.

« Top-down » inverse dynamics approach :

The use of the «Top-down» inverse dynamics approach with the double inverted pendulum model of human stance needs two steps and is illustrated on the figure A-1. 
In the following, masses and segment positions are assumed to be known. The first step consists in isolating the upper pendulum segment to bring out the force due to the lower limbs acting on the upper pendulum segment $F_{1 / 2}$ as well as the joint torque $u_{2}$ :

$\left\{\begin{array}{l}F_{1 / 2}=m_{2} a_{G_{2}}-P_{2} \\ u_{2}=M_{P_{2}, H}-I_{2} \ddot{\theta}_{2}\end{array}\right.$

After having computed the articular solicitations at the hip, the second step consists in isolating the lower pendulum segment constituted by the legs and the thighs. Then, the force acting from the feet to the lower pendulum $F_{0 / 1}$ as well as the ankle torque $u_{1}$ are:

$$
\left\{\begin{array}{l}
F_{0 / 1}=m a_{G_{1}}-F_{2 / 1}-P_{1} \\
u_{1}=-M_{P_{1}, 0}-M_{F_{2 / 1}, 0}+u_{2}+I_{1} \ddot{\theta}_{1}
\end{array}\right.
$$

\section{«Bottom-up » inverse dynamics approach:}

The use of the «Bottom-up » inverse dynamics techniques in the case of human standing modelled by a double inverted pendulum consists in two steps depicted in figure A-2.

The first step consists in isolating the feet to bring out the force due to the lower limbs acting on the feet $F_{0 / 1}$ as well as the ankle torque $u_{1}$ :

$$
\left\{\begin{array}{l}
F_{1 / 0}=m_{p} a_{p}-F_{e x t}-P_{p} \\
u_{1}=M_{P_{p}, O}+M_{F_{e x t}, O}-I_{0} \ddot{\theta}_{0}
\end{array}\right.
$$

After having computed the articular solicitations at the ankle, the second step consists in isolating the lower pendulum segment. Then, the force acting from the lower to the upper pendulum segment $F_{1 / 2}$ as well as the hip torque $u_{2}$ are:

$$
\left\{\begin{array}{l}
F_{2 / 1}=m a_{G_{1}}-F_{0 / 1}-P_{1} \\
u_{2}=M_{P_{1}, H}+M_{F_{0 / 1}, H}+u_{1}-I_{1} \ddot{\theta}_{1}
\end{array}\right.
$$


Assumption: The feet movements are small, quantities $m a_{p}$ and $I_{0} \ddot{\theta}_{0}$ are neglected and the feet are assumed to be fixed to the ground while quiet standing.

\section{REFERENCES}

Bouarar T, Guelton K, Manamanni N. LMI based H-infinity controller design for uncertain Takagi-Sugeno descriptors subject to external disturbances. 3rd IFAC Workshop on Advanced Fuzzy/Neural Control. Valenciennes, France, octobre 2007.

Bernal M, Husek P, Kucera V. Nonquadratic Stabilization of Continuous-Time Systems in the Takagi-Sugeno Form. Kybernetika 2006; 42(6):665-672.

Cahouët V, Luc M,Amarantini D. Static optimal estimation of joint accelerations for inverse dynamics problem solution. $\mathrm{J}$ of Biomechanics 2002; 35(11):1507-1513.

Cappozzo A, Leo T, Pedotti A. A general computing method for the analysis of human locomotion. J of Biomechanics 1975; 8:307-320.

Carpenter MG, Frank JS, Winter DA, Peysar GW. Sampling duration effects on centre of pressure summary measures. Gait \& Posture 2001; 13(1):35-40.

Challis JH, Kerwin DG. Quantification of the uncertainties in resultant joint moments computed in a dynamic activity. J of Sports Sciences 1996; 14:219-231.

Gahinet P, Nemirovski A, Laub AJ, Chilali M. LMI Control Toolbox for Use with MATLAB, The Mathworks Partener Series. 1995.

Guelton K. Estimation des caracteristiques du mouvement humain en station debout. Mise en œuvre d'observateurs flous sous forme descripteur. $\mathrm{PhD}$ thesis, Valenciennes University, France, 2003. 
Guelton K, Delprat S, Barbier F, Guerra TM, Armand S, Lepoutre FX. Global optimization of a double inverted pendulum model for the ankle and hip joint torques estimation in standing. 12th ESMAC congress, Gait \& Posture 2003, 18(2):80-123.

Guelton K, Delprat S, Guerra TM. Joint torques estimation in standing by mean of a fuzzy descriptor unknown inputs observer. 6th IFAC Symposium on Modelling and Control in Biomedical Systems, Reims, France, 20-22 September 2006.

Guerra TM, Guelton K, Delprat S. A class of nonlinear observers in descriptor form: LMI based design with application in biomechanics. 2nd IFAC Workshop on Advanced Fuzzy/Neural Control, Oulu, Finland, 2004.

Guerra TM, Kruszewski A, Vermeiren L, Tirmant H. Conditions of output stabilization for nonlinear models in the Takagi-Sugeno's form. Fuzzy Sets and Systems 2006; $157: 1248-1259$.

Gustafsson F. Determining the initial states in forward-backward filtering. IEEE Trans on Signal Processing 1996; 44:988-992.

Hatze $\mathrm{H}$. The fundamental problem of myoskeletal inverse dynamics and its implications. $\mathrm{J}$ of Biomechanics 2002; 35(1):109-115.

D. Khiar, Lauber J, Floquet T, Colin G, Guerra TM, Chamaillard Y. Robust TakagiSugeno fuzzy control of a spark ignition engine. Control Engineering Practice 2007, 15(12):1446-1456

Liu J. On-line soft sensor for polyethylene process with multiple production grades. Control Engineering Practice 2007, 15(7):769-778

Liu X, Zhang Q. New approach to $H_{\infty}$ controller designs based on fuzzy observer for T-S fuzzy systems via LMI. Automatica 2003; 39(9):1571-1582 
Marx B, Koenig D, Ragot J. Design of observers for Takagi-Sugeno descriptor systems with unknown inputs and application to fault diagnosis, IET Control Theory \& Applications 2007; 1(5):1487-1495

McCaw ST, De Vita P. Errors in alignement of center of pressure and foot coordinates affecte predictes lower extremity torques. J of Biomechanics 1995; 28:985-988.

Mitra SK. Digital Signal Processing, 2nd ed., McGraw-Hill Ed, 2001.

Nashner L, McCollum G. The organization of human postural movements: a formal basis and experimental synthesis. Behav Brain Sci 1985; 8:135-72

Nougier JP. Méthodes de calcul numérique. Vol 2: Fonctions équations aux dérivées. Ed. HERMES Science Europe, Paris, France, 2001.

Sala A, Guerra T M, Babuska R (2005) - Perspectives of fuzzy systems and control Fuzzy Sets \& Systems (Special Issue: $40^{\text {th }}$ Anniversary of Fuzzy Sets) - 153 (3), $432-444$

Takagi T, Sugeno M. Fuzzy identification of systems and its applications to modeling and control. IEEE Trans on System Man and Cybernetics 1985; 15 (1):116-132.

Tamer A and Malik OP. Identification of nonlinear systems by Takagi-Sugeno fuzzy logic grey box modeling for real-time control. Control Engineering Practice 2005, 13(12):14891498

Tanaka K, Wang HO. Fuzzy control systems design and analysis. A linear matrix inequalitiy approach. Willey, New York, 2001.

Taniguchi T, Tanaka K, Wang HO. Fuzzy descriptor systems and nonlinear model following control. IEEE Trans. on Fuzzy Systems 2000; 8(4):442-452.

Taniguchi T, Tanaka K, Wang HO. Model construction rule resduction and robust compensation for generalized form of Takagi-Sugeno fuzzy systems. IEEE Trans. on Fuzzy Systems 2001; 9(4):525-537. 
Tuan HD, Apkarian P, Narikiyo T, Yamamoto Y. Parameterized linear matrix inequality techniques in fuzzy control system design. IEEE Trans on Fuzzy Systems 2001; $9(2): 324-332$

Winter DA. Biomechanics and motor control of human movement. New York: Wiley; 1990.

Zajac FE. Muscle coordination of movement: a perspective. J of Biomechanics 1993; 26:109-124.

Zajac FE, Gordon ME. Determining muscle's force and action in multi-articular movement. Exercise and Sport Science Reviews 1989; 17:187-230. 


\section{Table and Figures:}

Fig. 1: A double inverted pendulum model of human standing.

Fig. 2: State variables and torques estimations following a simple trajectories simulation.

Fig. 3: Angular position and torques estimations following a complex trajectories simulation.

Fig. 4: Experimental devices.

Fig. 5: experimental results for the CTE experiment.

Fig. 6: Detailed ankle torques estimations from the CTE experiment

Fig. 7: Evolution of the fuzzy membership function $v_{1}$.

Fig. 8: "Top-down" and "Bottom-up" inverse dynamics approaches

Fig. 9: Comparison of inverse dynamics approaches and the fuzzy unknown inputs observer for the CTE experiment, (a) ankle torques estimations, (b) hip torques estimations Fig. 10: Residual torques at the hip due to the iterative inverse dynamics approaches

Fig. 11: Comparison of inverse dynamics approaches and the fuzzy unknown inputs observer for the QS experiment, (a) ankle torques estimations, (b) hip torques estimations Fig. A-1: «Top-down» inverse dynamics approach applied to the double inverted pendulum model of human standing.

Fig. A-2: «Bottom-up» inverse dynamics approach applied to the double inverted pendulum model of human standing.

Tab. 1: Human standing model parameters. 


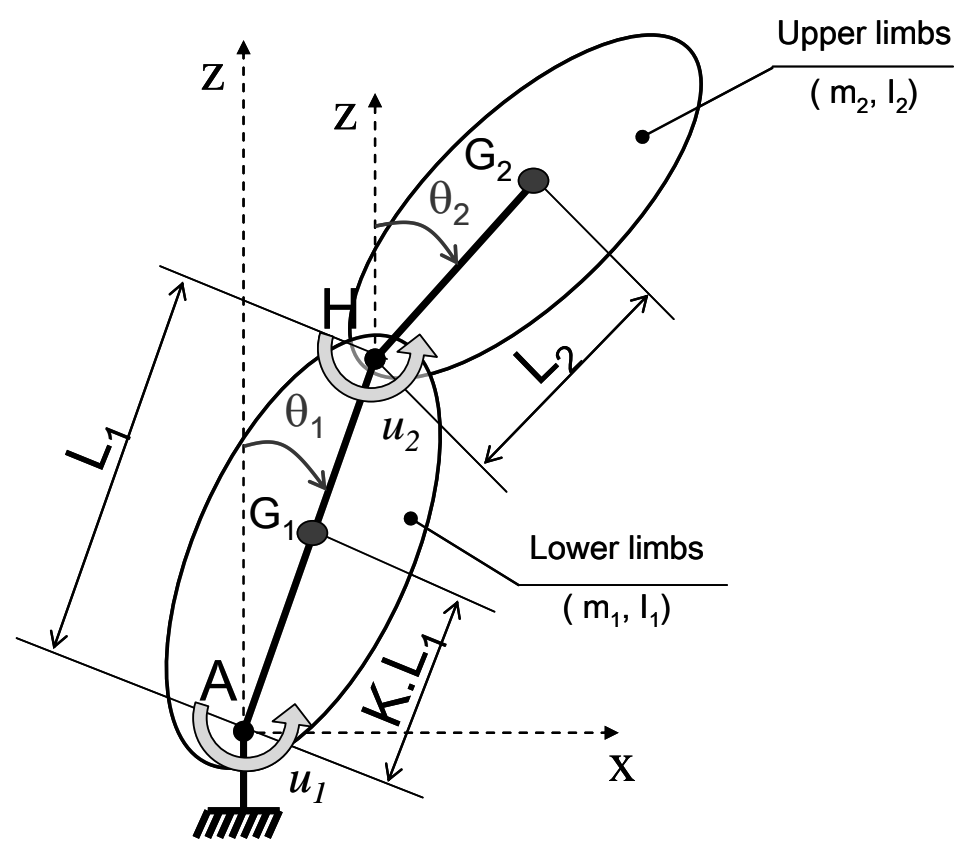

Fig. 1: Double inverted pendulum model of human standing.
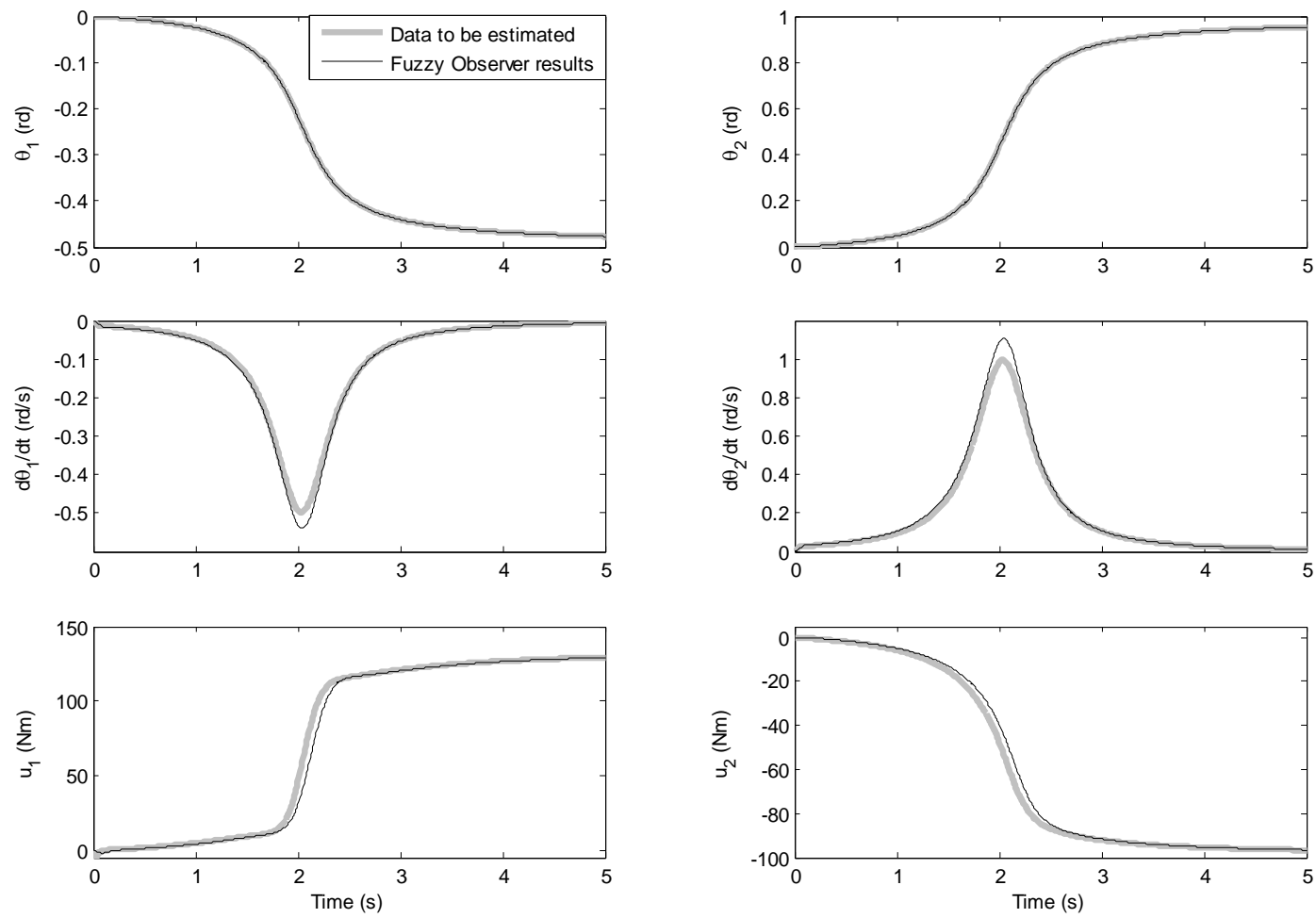

Fig. 2: State variables and torques estimations following a simple trajectories simulation. 

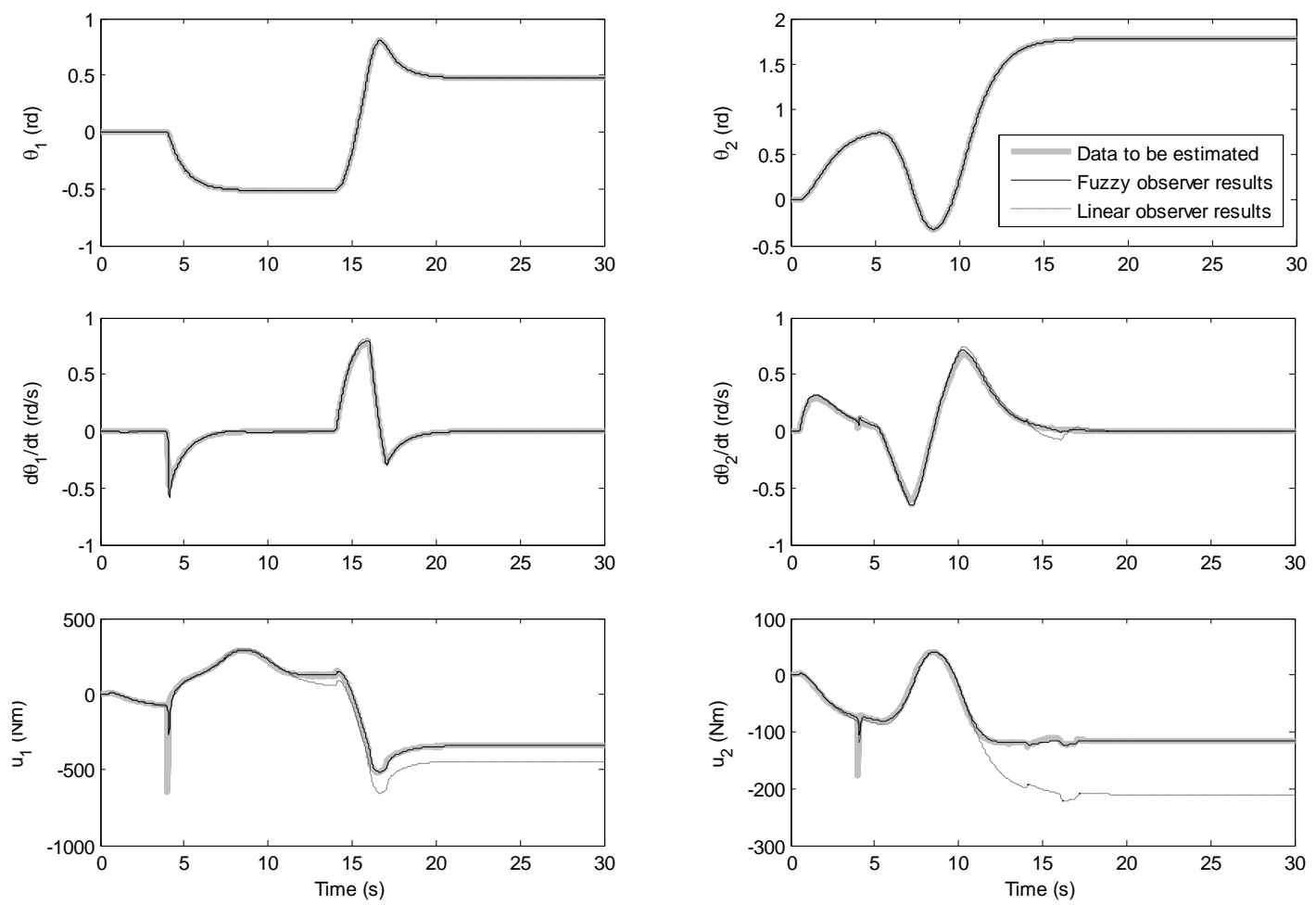

Fig. 3: Angular position and torques estimations following a complex trajectories simulation.

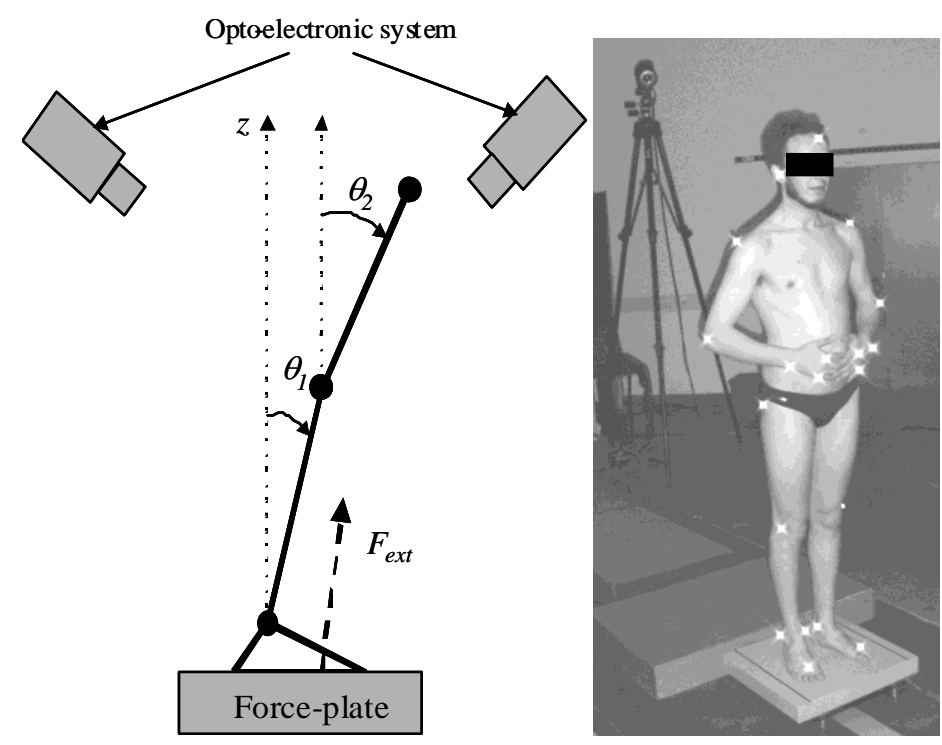

Fig. 4: Experimental devices. 

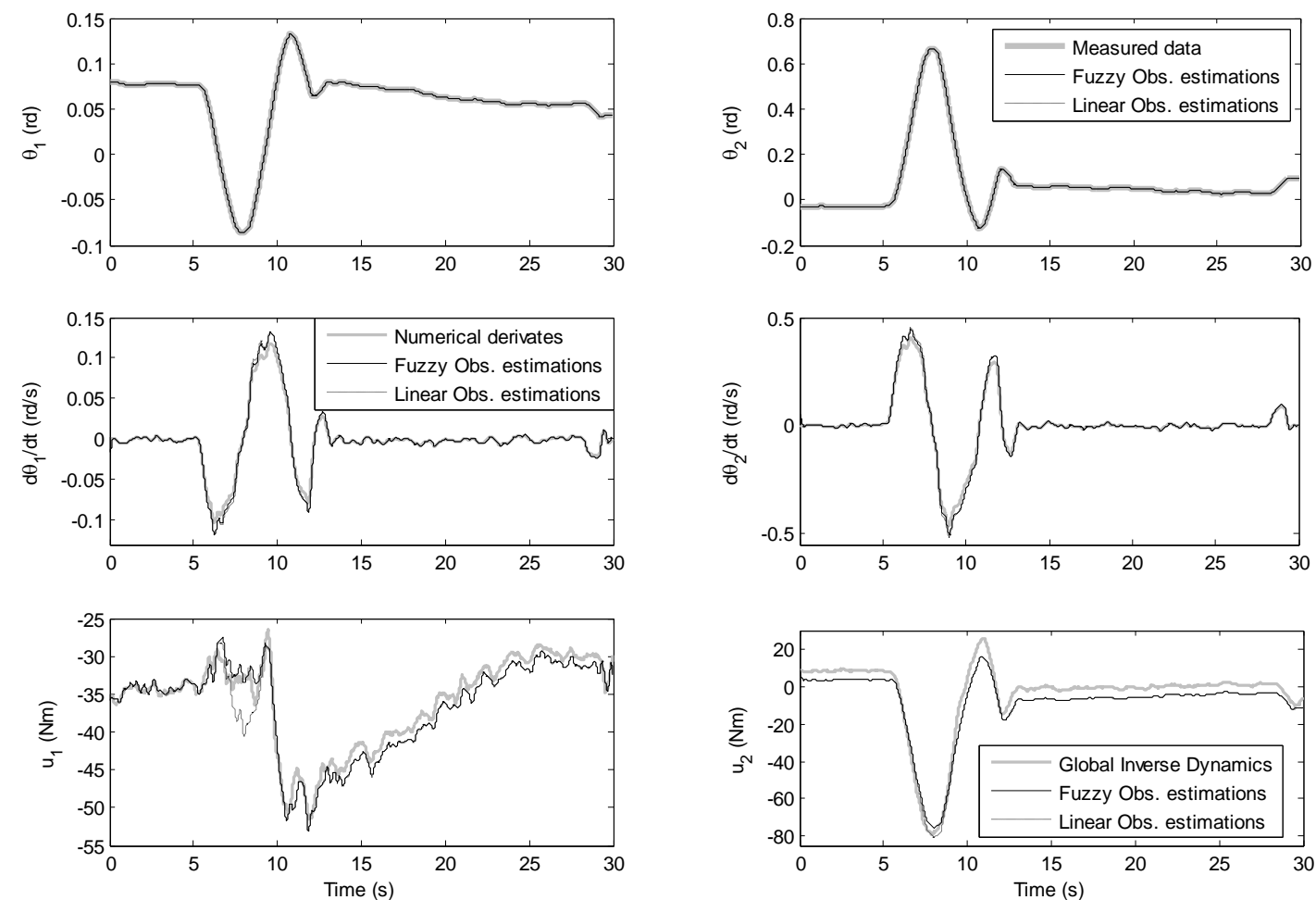

Fig. 5: experimental results for the CTE experiment.

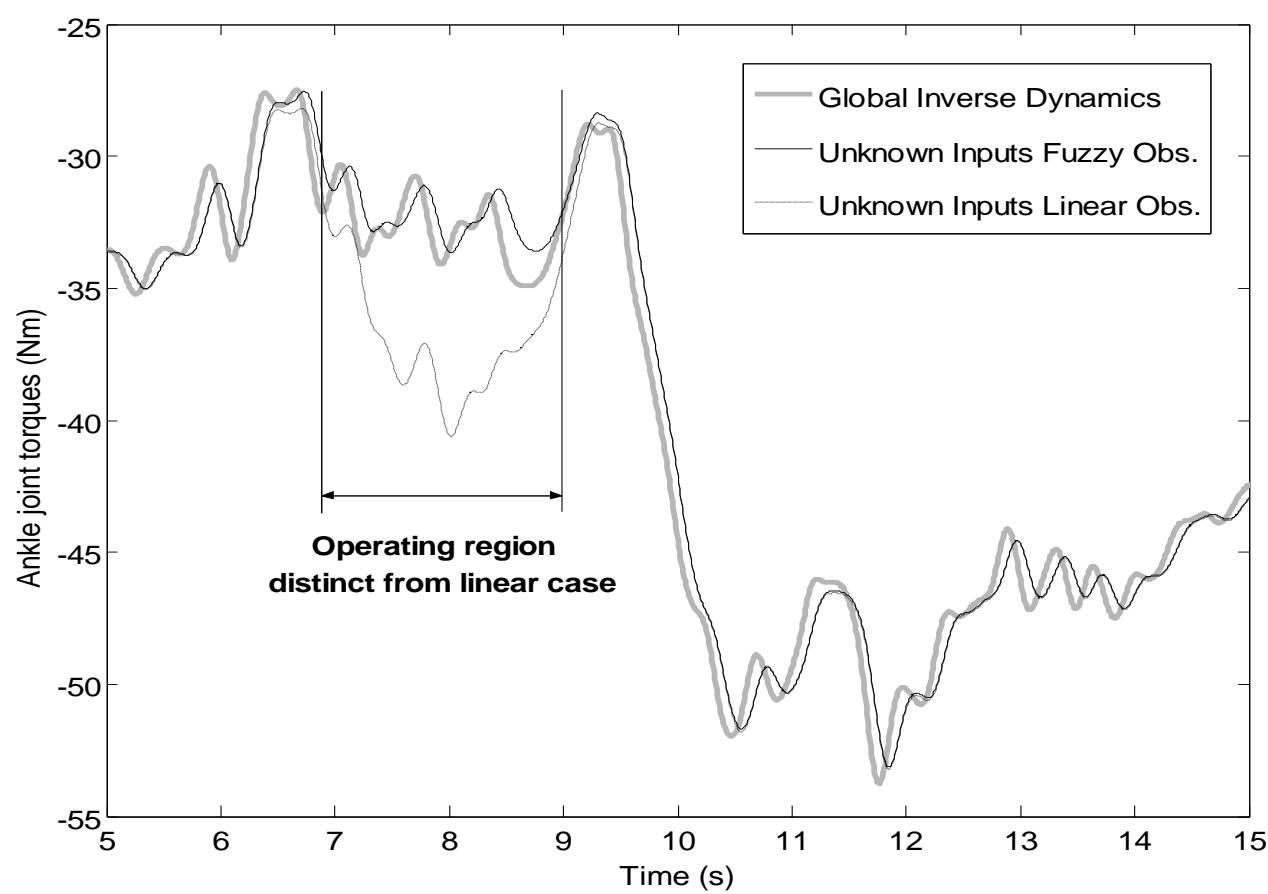

Fig. 6: Detailed ankle torques estimations from the CTE experiment 


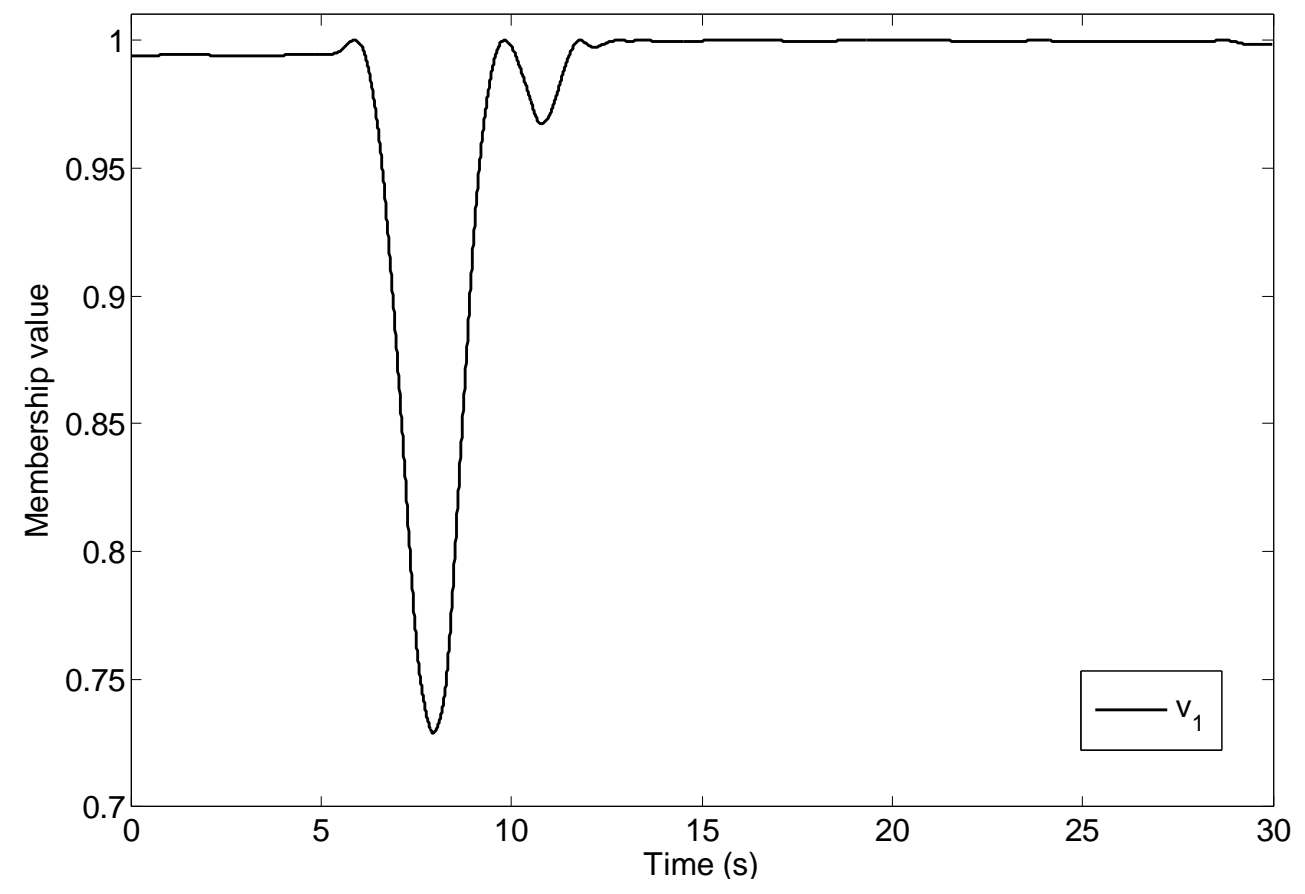

Fig. 7: Evolution of the fuzzy membership function $v_{1}(x(t))$.

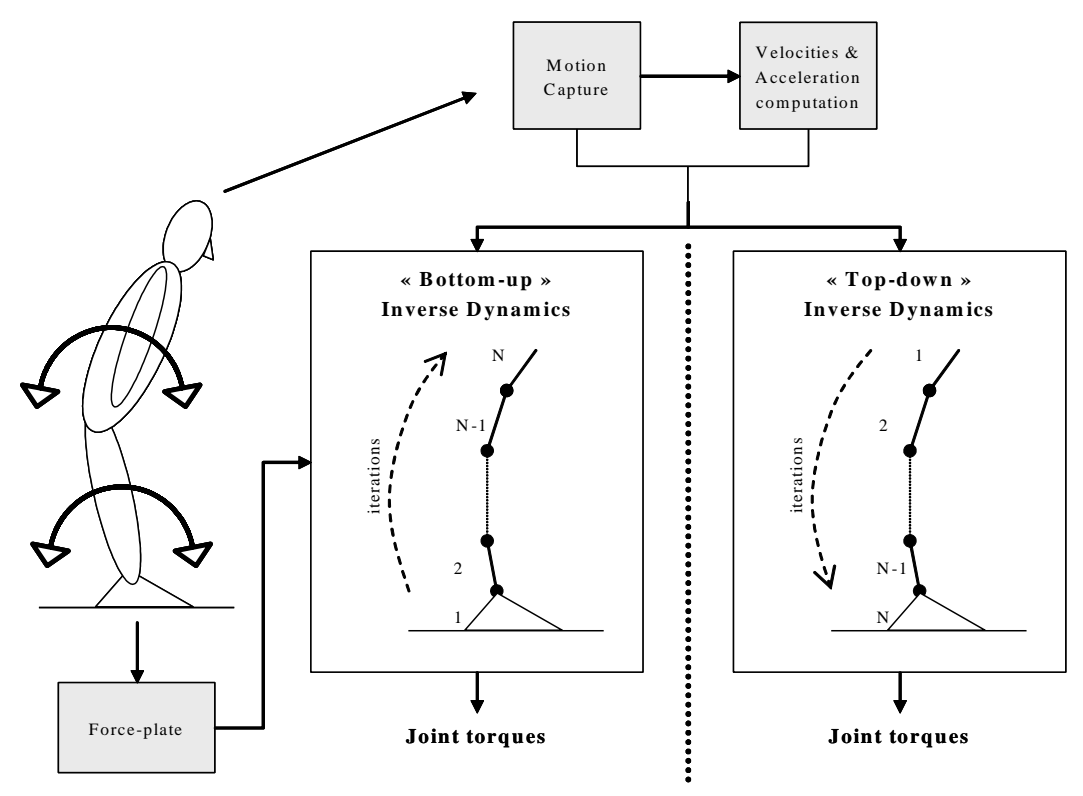

Fig. 8: "Top-down" and "Bottom-up" inverse dynamics approaches 

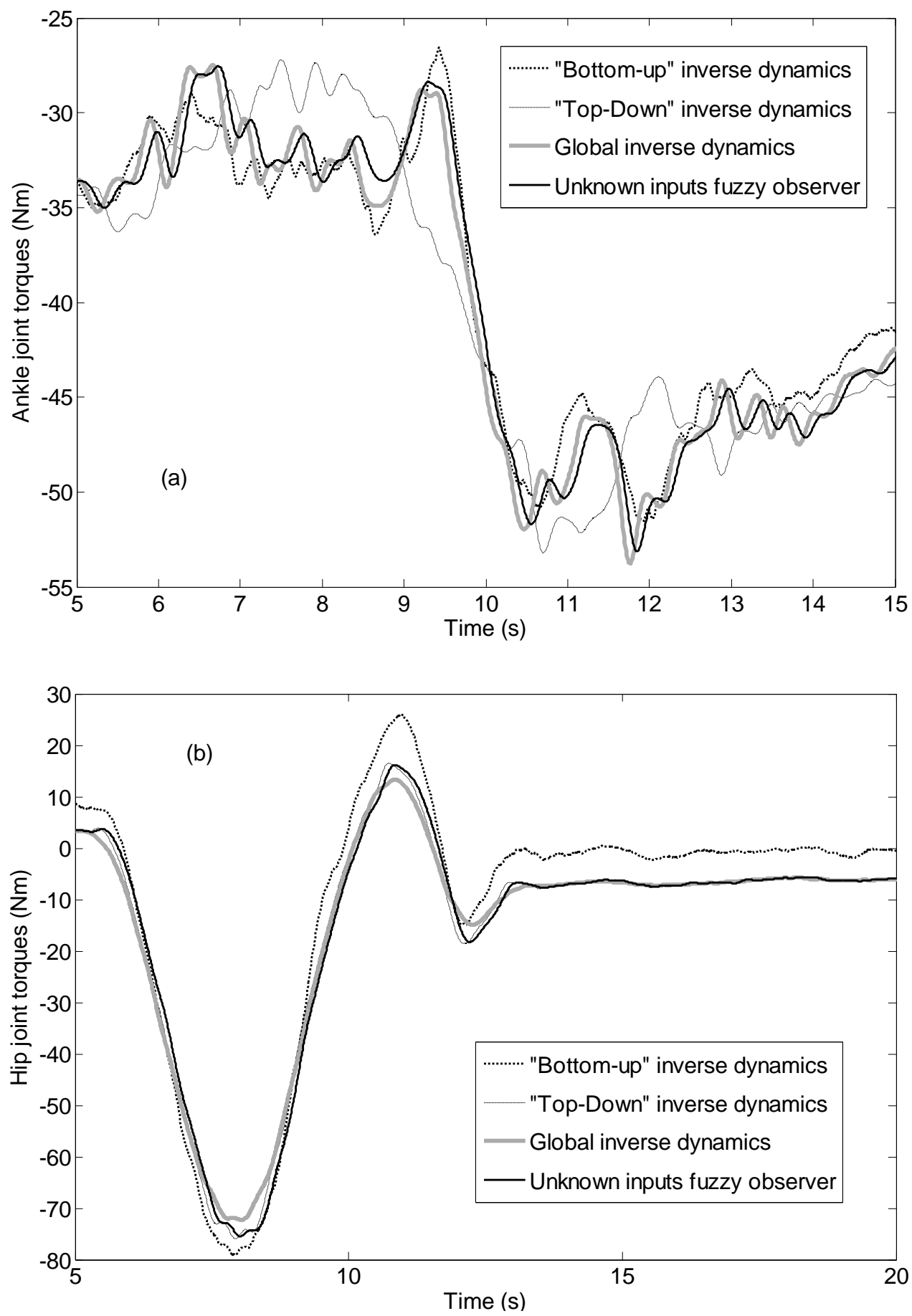

Fig. 9: Comparison of inverse dynamics approaches and the fuzzy unknown inputs observer for the CTE experiment, (a) ankle torques estimations, (b) hip torques estimations 


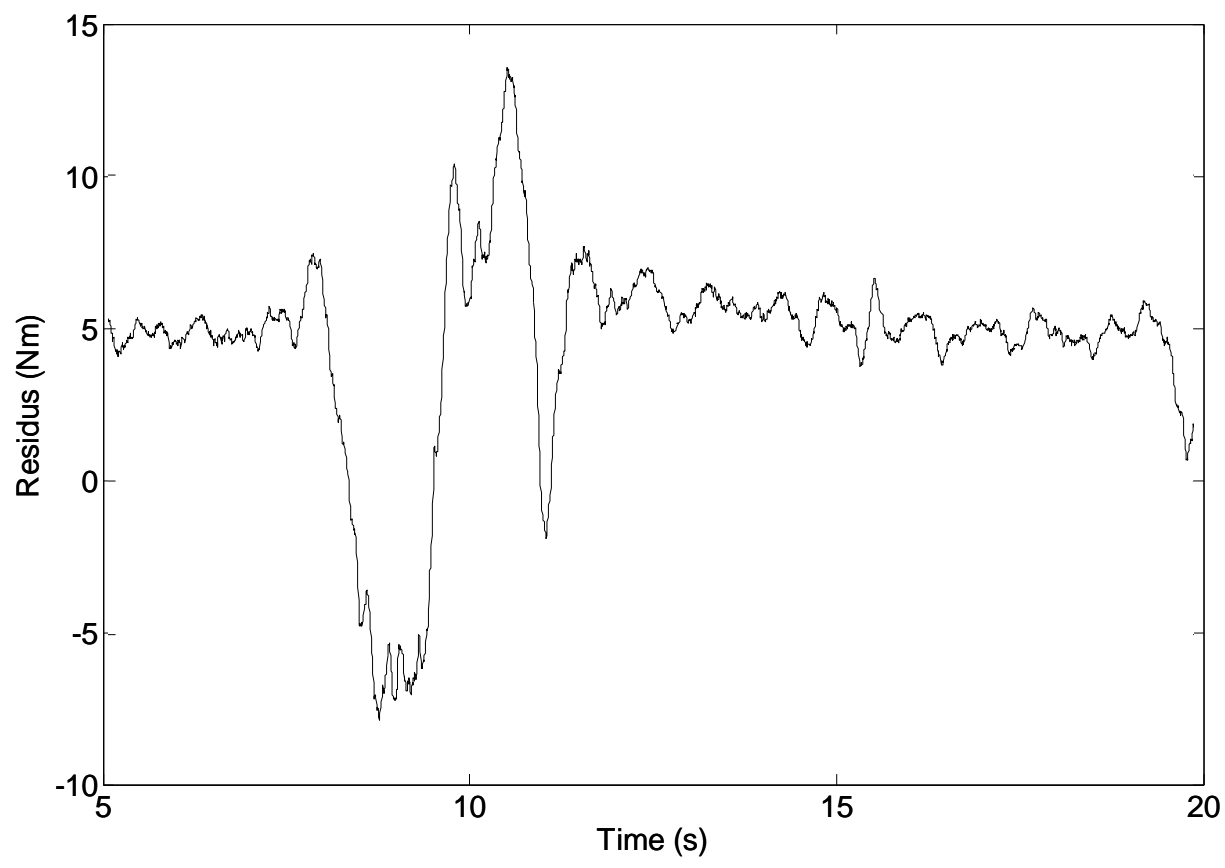

Fig. 10: Residual torques at the hip due to the iterative inverse dynamics approaches 

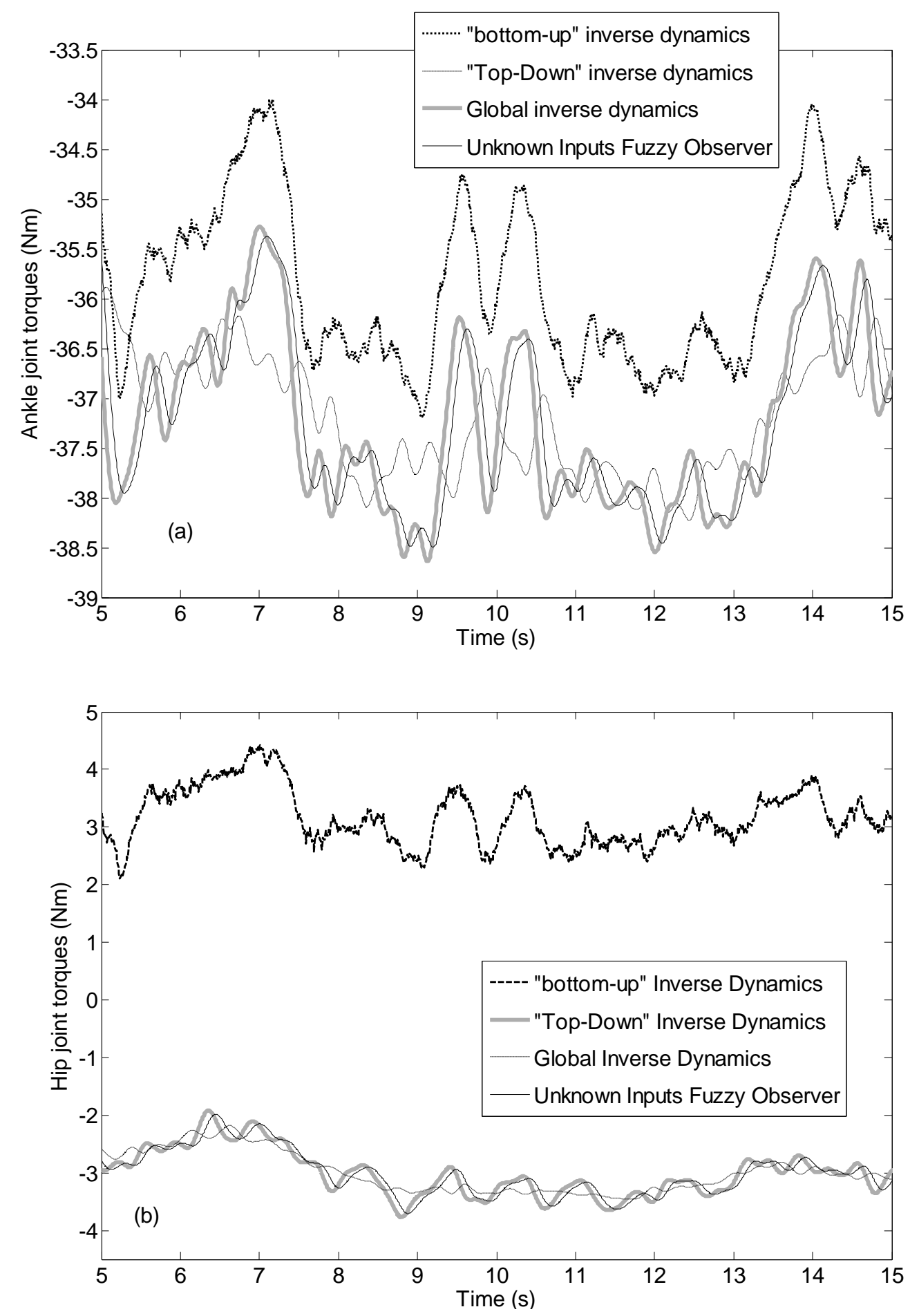

Fig. 11: Comparison of inverse dynamics approaches and the fuzzy unknown inputs observer for the QS experiment, (a) ankle torques estimations, (b) hip torques estimations 

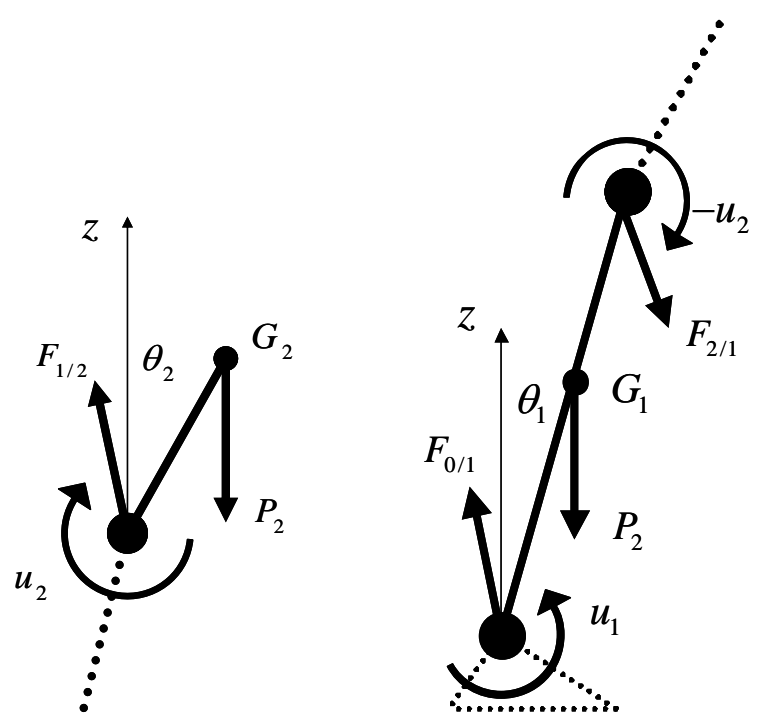

Fig. A-1 : «Top-down » inverse dynamics approach applied to the double inverted pendulum model of human standing.
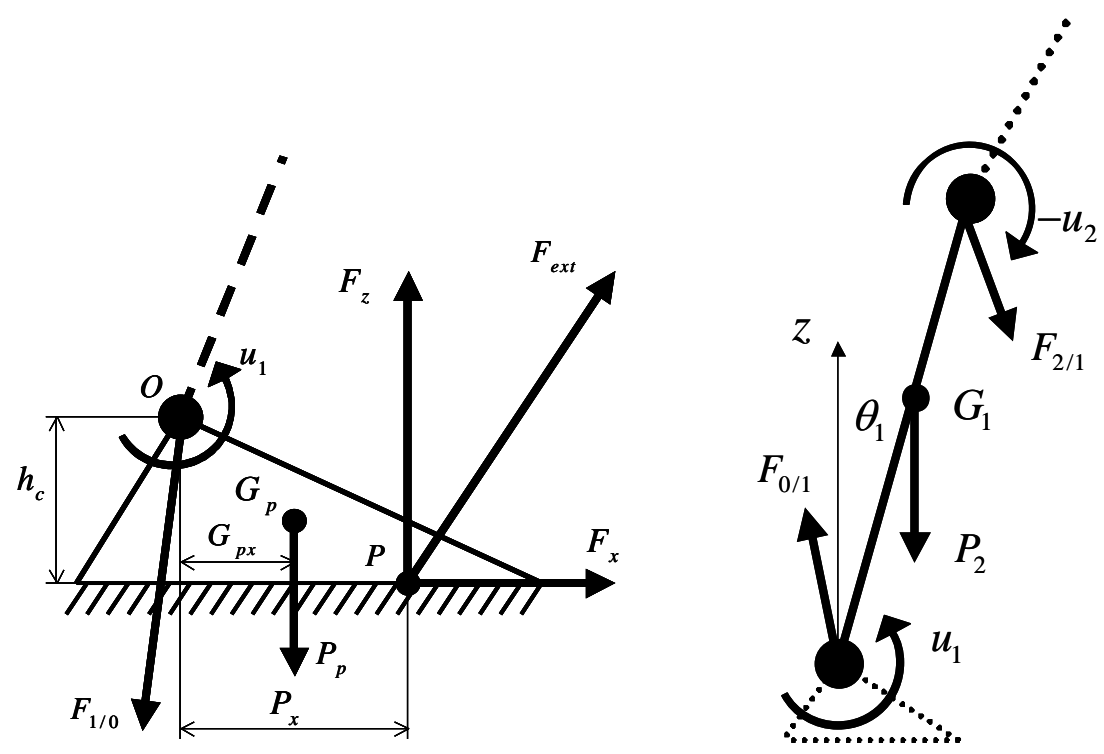

Fig. A-2: «Bottom-up » inverse dynamics approach applied to the double inverted pendulum model of human standing. 


\begin{tabular}{|c|c|l|}
\hline Notation & Value & designation \\
\hline$L_{1}$ & $0.87 \mathrm{~m}$ & Lower limbs length (without feet) \\
\hline$L_{2}$ & $0.26 \mathrm{~m}$ & $\begin{array}{l}\text { Distance between the hip (point } \mathrm{H} \text { ) and the upper } \\
\text { pendulum }\{\text { trunk+head+upper limbs }\} \text { centre of } \\
\text { gravity (point } \mathrm{G}_{2} \text { ) }\end{array}$ \\
\hline$I_{1}$ & $1.25 \mathrm{~kg} / \mathrm{m}^{2}$ & Lower limbs (without feet) ineretia \\
\hline$I_{2}$ & $2.32 \mathrm{~kg} / \mathrm{m}^{2}$ & $\begin{array}{l}\text { Upper pendulum }\{\text { trunk+head+upper limbs }\} \\
\text { ineretia }\end{array}$ \\
\hline$m_{1}$ & $21.87 \mathrm{~kg}$ & Lower limbs (without feet) mass \\
\hline$m_{2}$ & $45.87 \mathrm{~kg}$ & Upper pendulum \{trunk+head+upper limbs $\}$ mass \\
\hline$K$ & $\begin{array}{c}0.525 \\
\text { (without } \\
\text { units) }\end{array}$ & $\begin{array}{l}\text { Lower limbs (without feet) centre of mass } G_{1} \\
\text { location coefficient relating to the length of the } \\
\text { lower pendulum }\end{array}$ \\
\hline
\end{tabular}

Tab. 1: Human standing model parameters. 